\title{
Modeling and compensation of dynamic effects in camera-based position measurement
}

\author{
Edouard Laroche ${ }^{\mathrm{a}, 1}$, Shingo Kagami ${ }^{\mathrm{b}}$, Loïc Cuvillon ${ }^{\mathrm{a}}$ \\ ${ }^{a} I C U B E$ laboratory, Strasbourg University \& $C N R S$, France \\ ${ }^{b}$ Graduate School of Information Sciences, Tohoku University, Japan
}

\begin{abstract}
Cameras are used as position sensors for camera-based control or metrology, the goal being to estimate the position of some markers or features. When one of these markers has significant displacement over the exposure time, the measurement delivered by image processing cannot be considered as a sample of the actual trajectory as dynamic effects become effective. In this paper, models are proposed that allow to reproduce accurately the measurements provided by a camera. Various cases are considered: with full or partial exposure time; with global shutter mode or rolling shutter mode. An experimental evaluation in the global shutter case shows the accuracies of the models.

When the continuous-time trajectory of a device needs to be reconstructed, these accurate models of the camera-based position measurement can be used in order to improve the trajectory provided by the camera. A methodology based on a hybrid Kalman filter is proposed to solve this general issue. An experimental evaluation is provided for the global shutter case, based on real images inspired from the problem of heart motion reconstruction.
\end{abstract}

Keywords: Camera-based position measurement, visual servoing, Kalman filter, camera model, dynamic effects

\footnotetext{
*Corresponding author

Email address: laroche@unistra.fr (Edouard Laroche)
} 


\section{Introduction}

Vision systems with real-time image processing have been developed during the last decades and, in association with efficient image processing algorithms, are now able to provide measurements of the position of an object at relatively high frequency. Based on this measurement, specific control schemes have been developed for controlling robotic manipulators [1]. For instance, Andersson developed a robot playing ping-pong based on a $60 \mathrm{~Hz}$ stereo vision system [2]. In 2000, Nakabo et al. reached a frame rate of $1000 \mathrm{~Hz}$ in a vision servo loop [3].

In vision-based systems, the camera and the associated image-processing algorithm can be considered as a sensor that provides discrete-time (DT) measurements of the continuous-time (CT) image position of a marker or an object. When considering the imperfections of this sensor, one generally focuses on the geometrical aspects, i.e. the projection model whose parameters can be estimated from a calibration procedure [4]. This model is static and deals with the distortion in the projection on the camera plane. Concerning the dynamic effects, one generally considers that the measurement behaves as a sampler that delivers the position at given sampling instants.

When the object or the camera is moving and the displacements over an integration period of the image are significant, the image is altered and the object or the marker appears as a blur or a blob. Several authors have introduced methods that allow to take profit of this effect for the estimation of some features of the movement. Klein and Drummond use the blur effects to estimate the camera rotation [5] whereas Borrachi estimates its translation [6]. Some works also consider the rolling shutter effect: Liang et al. compensate the effects due to a translational motion [7]; Ait-Aider et al. rely on the deformation of lines to estimate the velocity [8].

Modeling the alteration of the measurement of the position of a marker, in the case of fast displacements, is a different subject. Due to the movement, the markers do not appear any more as simple disks but rather as more complex shapes, depending on the trajectory. Then, the measurement provided by the image processing differs from what would be provided by a pure sampler. Very few papers consider more complex models. Among them, Ranftl et al. used a simple discrete-time (DT) model of the camera for performing visual servoing for a fast ultrasonic actuator [9]. Following Luna et al., fast dynamics can be considered as a perturbation and one could try to attenuate them [10]. 
For reducing the blur effect, common sense recommends to reduce the exposure time. But this leads to images with less amplitude in the pixel values and makes the detection less accurate, particularly when sufficient luminance is not available. Therefore, we consider that there is a need for models reproducing this phenomenon.

The few attention devoted to the current subject can be attributed to the fact that in most of the applications, the DT nature of the measurement is the final information. For instance, in visual servoing, a DT model of the system with a speed reference as input and the DT measurement of the position provided by the camera can be identified and used for control, globally accounting for all the dynamics of the robotic system, including the camera. However, in some applications, the CT signal is of interest. For instance, Cuvillon et al. used a high-speed camera in order to reconstruct the CT trajectory of the heart [11]. Neglecting the camera dynamics, it was considered that a high-speed camera was necessary, with a frame rate higher than the heart-beating harmonics with some margin. Instead of selecting a high-speed camera, one can wonder if a more simple one could have been used in combination with an accurate model, therefore reducing the cost of the system. Notice that the reconstruction of the CT trajectory from the DT measurements provided by the camera requires a model. When such a model is available, the Kalman filter is an usual tool allowing to combine both model and measurement data in order to reconstruct the CT trajectory.

The Kalman filter has become a very common tool and is used in many fields including signal processing and robotics. Initially introduced for state and parameter estimation, it appeared to be a very flexible mean for the fusion of heterogeneous data. In its theoretical background, the noises affecting the system are assumed to be the realization of stochastic processes. However, in practice, the covariance matrices of these noises can be used as tuning parameters in order to obtain the proper behavior of the filter.

In robotics and metrology, the goal is often to reconstruct the 3D position of points or objects from a collection of 2D positions in images. Naturally, the evaluation of the effects of the camera on the 3D positions is of interest. However, the current paper focuses on the errors in the image. The models developed herein will be of interest for evaluating the impact on the 3D positions but this issue will not be considered in the current paper.

The scope of this paper is twofold. First, models of the camera-based position measurement (CBPM) are developed for different kinds of cameras. 
These models can be used to improve the simulation or to derive a more accurate model dedicated to control design. Second, a methodology is proposed for the estimation of the CT trajectory of a system where the measurement is made by a camera. This methodology is based on a Kalman filter that relies on a CBPM model, such as one of those presented in the first part of the work. The paper is organized as following. Section 2 presents the models of CBPM. An experimental comparison of several models is made in Section 3. The Kalman filter and the adaptations needed for the reconstruction of the CT trajectory from the DT measurement are presented in Section 4. An application for the reconstruction of a periodic trajectory is presented in Section 5. Section 6 concludes the paper.

This paper summarizes several conference papers. The model of a fullshutter mode camera has been initially considered in [12]. In [13], a model of the rolling-shutter mode has been proposed. In addition, this paper presented the evaluation setup and included experimental results for the global-shutter mode camera. In [14], the methodology for the estimation of the CT trajectory was proposed in addition to its use in the reconstruction of a periodic signal.

\section{Models of camera-based position measurement}

\subsection{Measurement of the position by camera}

A scene being equipped with visual markers, it is possible to measure their positions in the image by some image processing algorithm. This technique is classically used for processing visual-servoing of robots [15]. The measurements can be used directly in a $2 \mathrm{D}$ visual servoing scheme or can be used in order to reconstruct a 3D pose, allowing to derive 3D control. Markers are generally spots of high luminous intensity that can be detected easily. In the visible spectrum, they can be obtained by LEDs or projection of laser beams [16]. It is also possible to use a dark marker over a bright surface $[17,18]$. Invisible electromagnetic radiation are also commonly used, as in the Polaris system by NDI that estimates the 3D pose from the 2D position of 3 spheres with high reflexion property in the infrared.

Usually, the elements of interest are simple circular markers and the goal of image processing is to compute the coordinates of their centers of mass in the image. In order to limit the computation cost for real-time implementation, the image processing often makes use of simple operations (binarization, 
threshold and computation of the center of mass in several sub-images centered around the current position).

Let us consider one marker and let $q(t)=\left[\begin{array}{ll}x(t) & y(t)\end{array}\right]^{\mathrm{T}}$ denote the vector of the coordinates of its center in the camera image at time $t$. Images are acquired at the camera frame rate $f_{c}$. For Image $I_{k}$ obtained at time $t_{k}=$ $k T_{c}$ where $T_{c}=1 / f_{c}$, the vector of the coordinates of the marker center-ofmass is denoted $q_{m}(t)=\left[\begin{array}{ll}x_{m}(k) & y_{m}(k)\end{array}\right]^{\mathrm{T}}$. Let us neglect the noise due to the spatial discretization of the image. In a static case or if displacements are small over the exposure time, one has $q_{m}(k)=q\left(t_{k}\right)$. In the case of significant displacements over the integration period, the equality is not valid any more. In this paper, we deal with models allowing to compute the DT trajectory $\left(x_{m}(k), y_{m}(k)\right)$ from the CT trajectory $(x(t), y(t))$. Accounting for the dynamic effects induced by the camera, these models are of interest for simulation, identification and control purposes.

For illustration, the following experimental setup has been used: a rigid object containing seven light-emitting diodes (LED) used as markers is moved by a pan-tilt platform, as shown in Fig. 1. The images given by a grey-level camera (see Fig. 2) allow to distinguish the seven markers easily. In the right-hand image in Fig. 2, one can observe the effect of a significant motion over exposure time. The object has been used previously in order to estimate the movements of a beating heart [11].

Based on the analysis of the shape of the blur, more complex imageprocessing algorithms could be used in order to determine the position of the marker at a given time (for instance at the end of the exposure time). However, in order to limit the complexity of the algorithm for real-time implementation, the simple centre-of-mass computation is generally used in robotics.

$\mathcal{H}_{1}$ small marker size

$\mathcal{H}_{2}$ no intersection over the integration period

$\mathcal{H}_{3}$ high camera resolution

$\mathcal{H}_{4}$ constant speed amplitude over the integration period

Table 1: List of assumptions for the CBPM model 


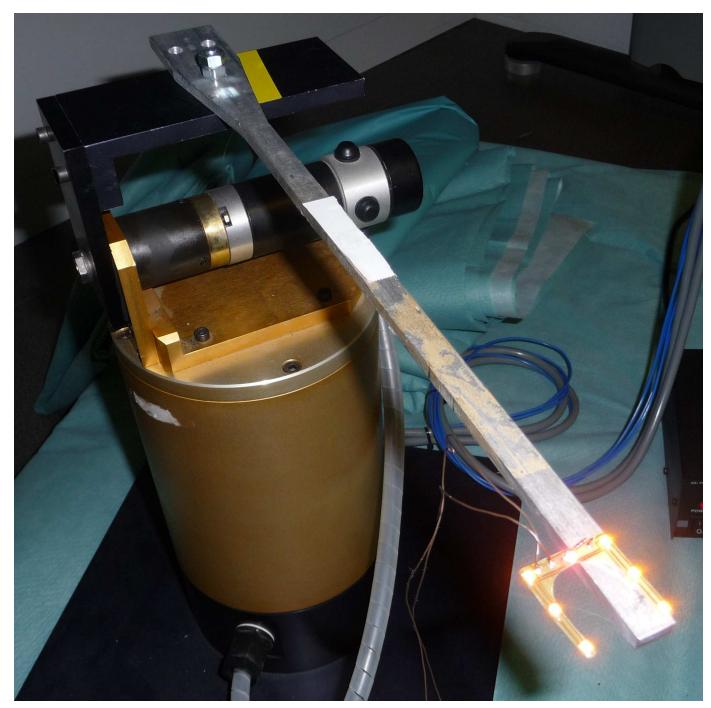

Figure 1: A pan-tilt robot with seven LEDs mounted on its tip

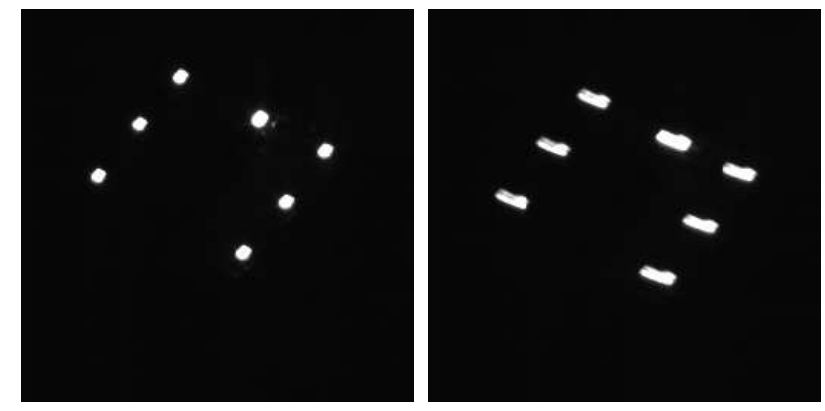

Figure 2: Two images of the 7-LED platform obtained by a grey-level camera at $25 \mathrm{~Hz}$. On the right-hand side, some significant displacement occur during exposure time. 


\subsection{Global shutter mode (GSM)}

\subsubsection{Nonlinear model}

In GSM, all the lines of image $I_{k}$ are acquired over the same time interval $\left[t_{k}-T_{e}, t_{k}\right]$ where $t_{k}=k T_{c}$ is the instant when the image is available and $T_{e}$ is the exposure time. Under Assumptions $\mathcal{H}_{1}-\mathcal{H}_{3}$ given in Tab. 1, the image provided by the displacement of an optical marker can be considered as a curve, as represented in Fig. 3. Then the center-of-mass measurement writes $q_{m}(k)=\frac{1}{l(k)} \int_{q\left(t_{k}-T_{e}\right)}^{q\left(t_{k}\right)} q \mathrm{~d} l$ where $\mathrm{d} l$ is an element of length of the trajectory and $l(k)=\int_{q\left(t_{k}-T_{e}\right)}^{q\left(t_{k}\right)} \mathrm{d} l$ is the length of the trajectory over $\left[t_{k}-T_{e} ; t_{k}\right]$. Considering that $\mathrm{d} l=\|\dot{q}\| \mathrm{d} t$, the model can be rewritten as a time-integral:

$$
q_{m}(k)=\frac{1}{l(k)} \int_{t_{k}-T_{e}}^{t_{k}} q\|\dot{q}\| \mathrm{d} t
$$

with $l(k)=\int_{t_{k}-T_{e}}^{t_{k}}\|\dot{q}\| \mathrm{d} t$. In the case of full exposure time $\left(T_{c}=T_{e}\right)$, this model results in the block-diagram of Fig. 4 where $\frac{1}{s}$ represents the integration over time. This model, called $\mathcal{M}_{1}$ in the sequel, is nonlinear and relies on three CT states gathered in $\tilde{X}_{b}(t)$ and three DT states gathered in $\tilde{X}_{c}(k)$.

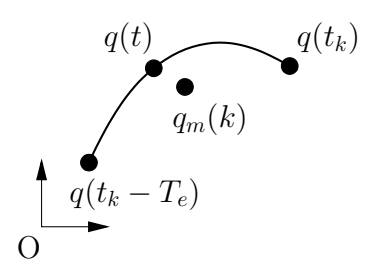

Figure 3: Simplified image obtained from displacement of a marker of small size over integration time

\subsubsection{Linear model}

Assuming that the speed modulus $\|\dot{q}\|$ remains constant over the integration period ( $\mathcal{H}_{4}$ in Tab. 1$)$, the model can be simplified into:

$$
q_{m}(k)=\frac{1}{T_{e}} \int_{t_{k}-T_{e}}^{t_{k}} q(t) \mathrm{d} t
$$

This model, called $\mathcal{M}_{2}$ in the sequel, is linear. It relies on two CT states and two DT states. A corresponding block-diagram is given in Fig. 5 for the full-exposure-time case. 


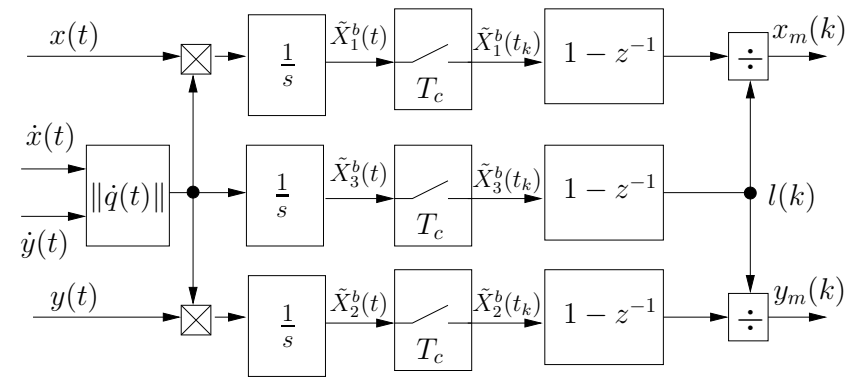

Figure 4: Model $\mathcal{M}_{1}$ : nonlinear model of a camera with global shutter mode and full exposure time (sampling of the CT signal at frequency $f_{c}=1 / T_{c}$ )

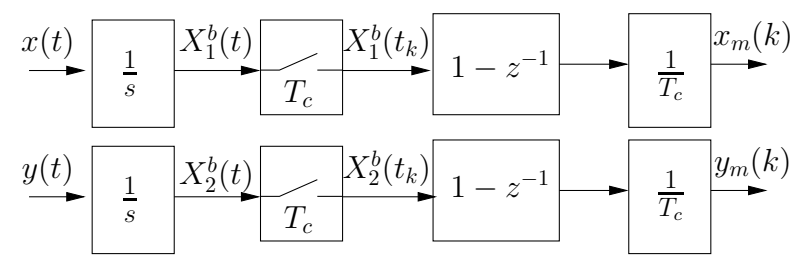

Figure 5: Model $\mathcal{M}_{2}$ : Linear model of a camera with global shutter mode and full exposure time

Model $\mathcal{M}_{2}$ has the advantage of its simplicity but relies on one more assumption than $\mathcal{M}_{1}$. Notice that, for constant velocity, they provide the same outputs.

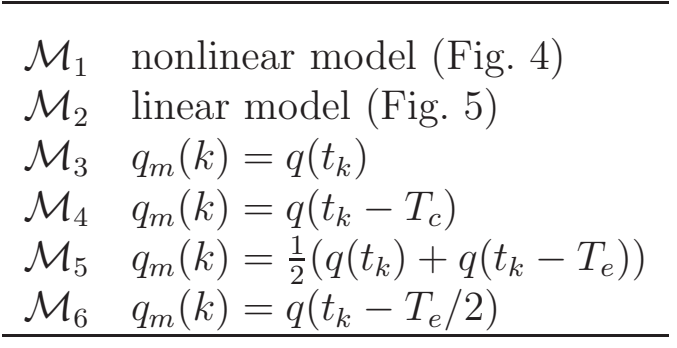

Table 2: List of models

\subsubsection{Other models}

Other more simple models can be used in order to predict the DT measurements from the CT trajectory. These models will be used in the sequel for comparison: 


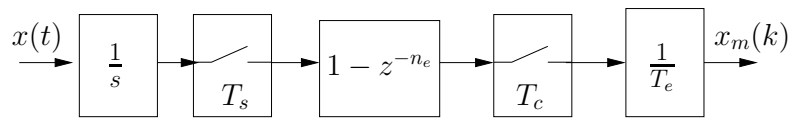

Figure 6: Model of a camera with GSM and partial exposure time (derived from $\mathcal{M}_{2}$ )

- The dynamic effects of the camera can be simply neglected, therefore considering the camera as a simple sampler, leading to $q_{m}(k)=q\left(t_{k}\right)$ $\left(\operatorname{model} \mathcal{M}_{3}\right)$ or $q_{m}(k)=q\left(t_{k-1}\right)\left(\operatorname{model} \mathcal{M}_{4}\right)$.

- The measurement can be approximated by the sum of the measurements provided by $\mathcal{M}_{3}$ and $\mathcal{M}_{4}$, leading to $q_{m}(k)=\frac{1}{2}\left(q\left(t_{k}\right)+q\left(t_{k-1}\right)\right)$ (model $\mathcal{M}_{5}$ proposed in [9]).

- The effects of the camera can be assumed as a pure delay of $T_{c} / 2$, leading to $q_{m}(k)=q\left(t_{k}-T_{c} / 2\right)\left(\operatorname{model} \mathcal{M}_{6}\right)$.

The different models are listed in Tab. 2.

\subsubsection{Partial exposure time}

The previous models can be modified by accounting for partial exposure time, i.e. when the exposure time $T_{e}$ is less than the camera period $T_{c}$. Assume that $T_{e} / T_{c}$ is rational and consider a sample time $T_{s}$ and integers $n_{c}$ and $n_{e} \leq n_{c}$ such that $T_{c}=n_{c} T_{s}$ and $T_{e}=n_{e} T_{s}$. For simplicity, the model derived hereafter from $\mathcal{M}_{2}$ is developed for coordinate $x$ but is also valid for $y$. Let us denote:

$$
J_{x}(t)=\int_{0}^{t} x(\tau) \mathrm{d} \tau
$$

One has:

$$
x_{m}(k)=\frac{1}{T_{e}}\left(J_{x}\left(k n_{c} T_{s}\right)-J_{x}\left(\left(k n_{c}-n_{e}\right) T_{s}\right)\right)
$$

A block diagram version of the corresponding model is represented in Fig. 6. It includes an integrator $\frac{1}{s}$, a sampler at $T_{s}$, a differentiator $1-z^{-n_{e}}$ sampled at $T_{s}$ and a second sampler at $T_{c}$. Applying the same modification in the block-diagram of Fig. 4, it is possible to derive a nonlinear model that accounts for partial exposure time for model $\mathcal{M}_{1}$.

\subsection{Rolling shutter mode (RSM)}

The paper is mostly devoted to GSM cameras that are more usual than RSM. However, as shown in this section, compact models can be also developed for RSM. 
In RSM, the lines of an image are acquired one by one with a delay. Consider that the image is composed of $n_{l}$ lines $L_{l}$ numbered from 0 to $n_{l}-1$. Lines are acquired in increasing order, so that an image is available as soon as $L_{n_{l}-1}$ is available. Denoting $t_{k}=k T_{c}$ the time at which Image $I_{k}$ is available and denoting $T_{d}$ the delay between the acquisitions of the first and the last lines, Line $L_{l}$ is acquired over $\left[t_{k}-T_{e}-\frac{n_{l}-l-1}{n_{l}-1} T_{d}, t_{k}-\frac{n_{l}-l-1}{n_{l}-1} T_{d}\right]$. See Fig. 7 for an illustration.

Neglecting the spatial discretization by considering a camera with infinite resolution, a simplified model can be derived. Consider that $y$ varies over $[0, \bar{y}]$. At time $t$, Image $I_{k}$ is affected by a point $(x(t), y(t))$ if the following inequalities are satisfied:

$$
\frac{\bar{y}}{T_{d}}\left(t+T_{d}-t_{k}\right) \leq y(t)<\frac{\bar{y}}{T_{d}}\left(t+T_{d}+T_{e}-t_{k}\right)
$$

Let $C_{k}(t)$ be a membership function for Image $I_{k}: C_{k}(t)=1$ if $(5)$ is satisfied and $C_{k}(t)=0$ otherwise. The residence time $T_{r}(k)$ for Image $I_{k}$ is the length of time over which the marker contributes to the image ${ }^{1}$ and writes

$$
T_{r}(k)=\int_{0}^{\infty} C_{k}(t) \mathrm{d} t
$$

Considering the previous assumptions, the measurement obtained from Image $I_{k}$ is given by:

$$
\begin{aligned}
x_{m}(k) & =\frac{1}{T_{r}(k)} \int_{0}^{\infty} C_{k}(t) x(t) \mathrm{d} t \\
y_{m}(k) & =\frac{1}{T_{r}(k)} \int_{0}^{\infty} C_{k}(t) y(t) \mathrm{d} t
\end{aligned}
$$

Directly implementing this model is not convenient as it would require the computation of many integrals (as many as the number of images). In the

\footnotetext{
${ }^{1}$ In the GSM, the residence time is constant and equal to the exposure time. In the RSM, this quantity is variable, depending on the displacement of the marker. The maximum residence time is $T_{e}+T_{d}$ and can be obtained for $\dot{y}=-\bar{y} /\left(T_{e}+T_{d}\right)$ (see in Fig. 7 from point $\left(t_{k}-T_{d}-T_{e}, \bar{y}\right)$ to $\left.\left(t_{k}, 0\right)\right)$. The minimum residence time can reach zero if $T_{d} \geq T_{e}$ and would be obtained with a marker starting at the bottom of the image $(y=0)$ at $t=t_{k}-T_{e}$ and moving towards the top of the image with an infinite speed $\dot{y}$. For $T_{d}<T_{e}$, the minimum residence time is $T_{e}-T_{d}$.
} 


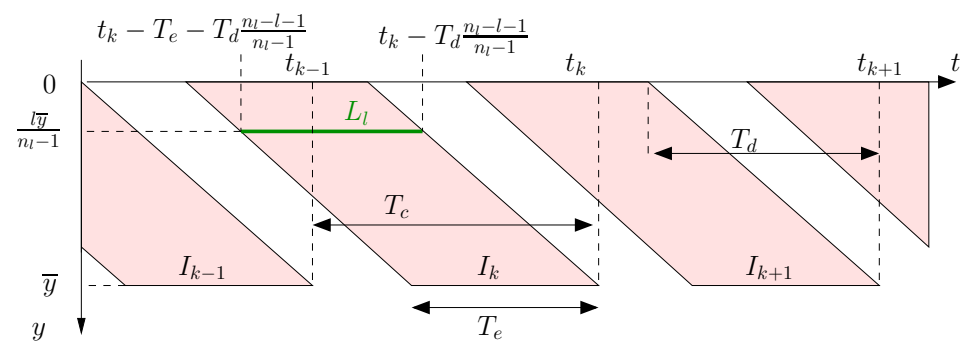

Figure 7: Explicative time diagram for the rolling shutter mode

sequel of this subsection, a more convenient formulation relying on a limited number of state variables is given in the remainder of the subsection.

One very specific feature of the RSM, is that several images have to be considered at a given time, as soon as $T_{e}+T_{d}>T_{c}$. In practice, a maximum of two images have to be considered as only one row can be readout at a time in an ordinary camera, yielding $T_{d} \leq T_{c}$. However, the theoretical maximum number of images writes $n_{i}=\left\lceil\frac{T_{e}+T_{d}}{T_{c}}\right\rceil$ ( $\lceil\cdot\rceil$ being the ceil function) and the model will be presented in the most general form.

Depending on the value of $y(t)$, the marker contributes to Image $I_{k+j}$, with $j=0, \ldots, n_{i}-1$, if $C_{k+j}(t)=1$. Based on this observation, let $I_{x j}$ and $I_{y j}, j=0, \ldots, n_{i}-1$, denote the $2 n_{i}$ state variables corresponding to coordinates $x$ and $y$. The state equations write:

$$
\begin{aligned}
& \dot{I}_{x j}(t)=C_{k+j}(t) x(t) \\
& \dot{I}_{y j}(t)=C_{k+j}(t) y(t)
\end{aligned}
$$

The measurement obtained from Image $I_{k}$ is finally the sum of the $n_{i}$ contributions of different vertical strips. For the latest strip, the contribution over $\left[t_{k-1}, t_{k}\right]$ obtained form $(7)$ writes $I_{x 0}\left(t_{k}\right)-I_{x 0}\left(t_{k-1}\right)$. The second earlier strip contributes over $\left[t_{k-2}, t_{k-1}\right]$ with $I_{x 1}\left(t_{k-1}\right)-I_{x 1}\left(t_{k-2}\right)$ and so on if more strips were involved. Using equations (7) to (10), the measurements are finally given by:

$$
\begin{aligned}
x_{m}(k) & =\frac{1}{T_{r}(k)} \sum_{j=0}^{n_{i}-1} I_{x j}\left(t_{k-j}\right)-I_{x j}\left(t_{k-j-1}\right) \\
y_{m}(k) & =\frac{1}{T_{r}(k)} \sum_{j=0}^{n_{i}-1} I_{y j}\left(t_{k-j}\right)-I_{y j}\left(t_{k-j-1}\right)
\end{aligned}
$$



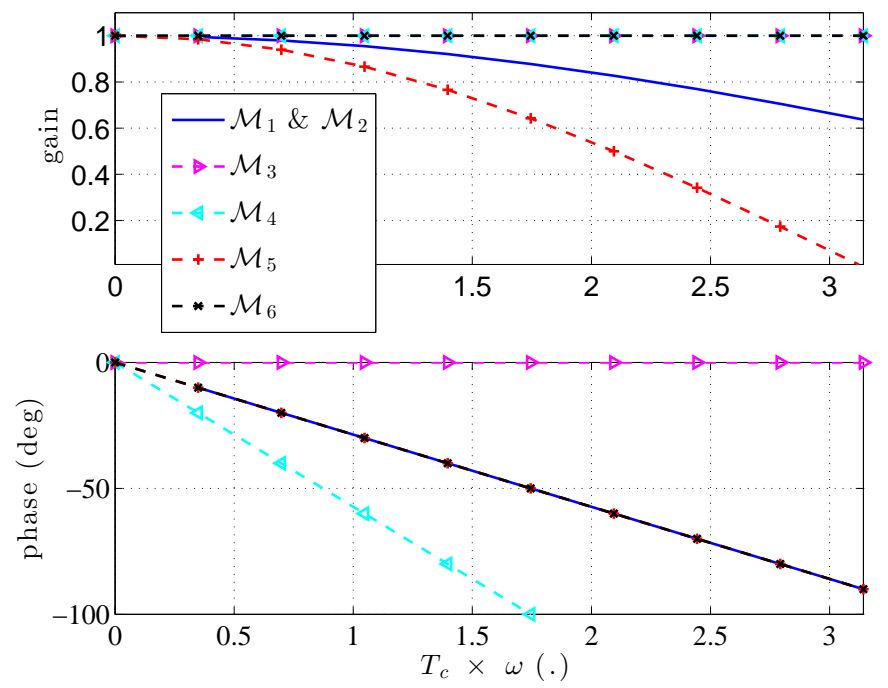

Figure 8: Bode Diagrams of the different models

The residence time $T_{r}(k)$ can be computed simultaneously with additional state variables. Let $\tau_{0}$ denote the time length corresponding to the current image, $\tau_{1}$ the time length corresponding to the next image and more generally $\tau_{j}, j=0, \ldots, n_{i}-1$, the time length corresponding to the $j^{\text {th }}$ future image. These states are updated according to the active mode:

$$
\dot{\tau}_{j}(t)=C_{k+j}(t)
$$

Finally, the residence time is computed by:

$$
T_{r}(k)=\sum_{j=0}^{n_{i}-1} \tau_{j}\left(t_{k}\right)-\tau_{j}\left(t_{k-1}\right)
$$

The resulting nonlinear model is composed of $3 n_{i}$ states $\left(I_{x j}, I_{y j}\right.$ and $\tau_{j}$, $\left.j=1, \ldots, n_{i}-1\right)$ and as many state equations $((9),(10)$ and $(13))$.

Notice that only the GSM is considered in the next sections of the paper. However, as they rely on the same hypotheses (Tab. 1), one can imagine that the GSM and RSM versions of $\mathcal{M}_{2}$ have similar accuracies.

\subsection{Frequency and time-domain evaluation}

Frequency-domain evaluation. Considering the DT-CT equivalence of the advance operator $z=\exp \left(T_{c} s\right)$, it is possible to compute the frequency response 

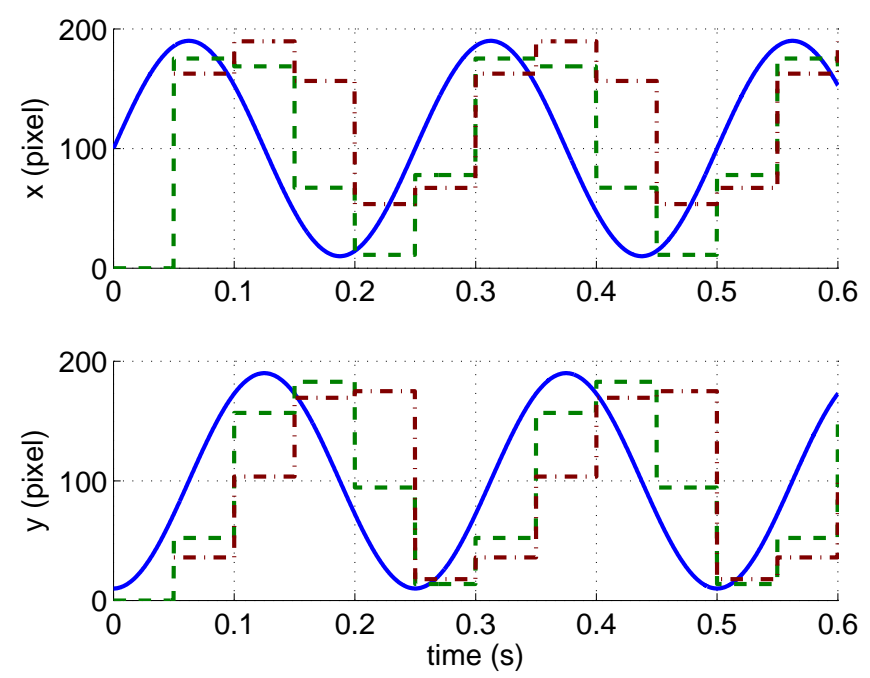

Figure 9: Simulation results of GSM and RSM models for a circular trajectory (plain: original CT trajectory, dash: GSM, dot/dash: RSM)

of the CBPM with full exposure time. For instance, for model $\mathcal{M}_{2}$, the equivalent CT model writes $\frac{1-\exp \left(-T_{c} s\right)}{T_{c} s}$. With $s=j \omega, \omega$ being the angular frequency, the frequency-domain behavior of the different models are given in Fig. 8. One can notice that the models differ either in gain or phase. Models $\mathcal{M}_{3}, \mathcal{M}_{4}$ and $\mathcal{M}_{6}$ have the same constant gain. Models $\mathcal{M}_{1}, \mathcal{M}_{2}, \mathcal{M}_{5}$ and $\mathcal{M}_{6}$ have the same phase.

Time-domain evaluation. Camera models can be directly implemented for simulation from the models given in the previous subsection ${ }^{2}$. For illustration, the models of the GSM and RSM ( $\mathcal{M}_{2}$ and its adaptation for RSM) were simulated with a circular trajectory at $4 \mathrm{~Hz}$ centered in a $200 \times 200$ pixel image. The camera period is $T_{c}=50 \mathrm{~ms}$ and the exposure time is $20 \mathrm{~ms}$. The GSM is implemented with $T_{s}=10 \mathrm{~ms}, n_{e}=2$ and $n_{c}=5$. For the RSM, a delay $T_{d}=T_{c}=50 \mathrm{~ms}$ is considered. The original CT trajectory and the DT trajectories obtained with the two shutter modes are given in Fig. 9. One can notice that the measurement obtained with GSM has a constant delay whereas the measurement obtained with RSM has a variable delay that in-

\footnotetext{
${ }^{2}$ A library with the Simulink models is available on the first author academic web pages (presently http://eavr.u-strasbg.fr/ laroche/camera/)
} 
creases with $y$ position. When the CBPM system is implemented on a plant to be simulated, the camera model is simply put at the end of the plant, such as in the block-diagram presented in Fig. 10.a.

\subsection{Derivation of a control-oriented model}

A DT model of the process, to be used for control or observation, can be obtained as following. Consider a process to be controlled equipped with a camera providing the output to be controlled $Y(k)$ thanks to a DT control signal $u(k)$ given to a CT process through zero-order hold $(\mathrm{ZOH})$ as shown in Fig. 10.a where $v(k)$ and $w(k)$ are noise signals that are neglected at this step.

Considering a linear CT model $G(s)$ of the process in the Laplace domain and choosing the linear model $\mathcal{M}_{2}$ for CBPM, a simplified model can be obtained such as in Fig. 10.b. The DT model can be obtained using a CT to DT conversion of $G(s) / s$, assuming that $u(t)$ comes from a $\mathrm{ZOH}$, and then multiplying the result by DT differentiator $\frac{z-1}{z T_{c}}$. It can be also obtained using a direct CT to DT transform based on a causal first-order hold assumption. Indeed, the camera has the same effect as an increase in the order of the hold. The resulting DT model is then obtained by an usual formula [19]:

$$
G(z)=\frac{(z-1)^{2}}{T_{c} z^{2}} \mathcal{Z} \circ \mathcal{L}^{-1}\left(\frac{G(s)}{s^{2}}\right)
$$

where $\mathcal{L}^{-1}$ is the inverse Laplace transform and $\mathcal{Z}$ is the Z-transform applied to the signal sampled at period $T_{c}$. A minimal realization is finally derived by canceling the poles and zeros at $z=1$.

Including the noise input $w$, the MIMO case with $n_{u}$ inputs $u, n_{w}$ inputs $w$ and $n_{q}$ measurements can be formulated in state space, provided that a state-space (SS) realization of $G(s)$ of order $n_{G}$ is available:

$$
\begin{aligned}
\dot{X}_{a} & =A_{G} X_{a}+B_{G 1} u+B_{G 2} w \\
q & =C_{G} X_{a}+D_{G 1} u+D_{G 2} w
\end{aligned}
$$

The augmented CT model $G_{a b}(s)$ including the CT states $X_{b}(t)$ of the camera model can be written with the following state-space realization:

$$
\left[\begin{array}{c}
\dot{X}_{a} \\
\dot{X}_{b} \\
\hline X_{b}
\end{array}\right]=\left[\begin{array}{cc|cc}
A_{G} & O_{n_{G} \times n_{q}} & B_{G 1} & B_{G 2} \\
C_{G} & O_{n_{q} \times n_{q}} & D_{G 1} & D_{G 2} \\
\hline O_{n_{q} \times n_{G}} & I_{n_{q} \times n_{q}} & O_{n_{q} \times n_{u}} & O_{n_{q} \times n_{w}}
\end{array}\right]\left[\begin{array}{c}
X_{a} \\
X_{b} \\
\hline u \\
w
\end{array}\right]
$$



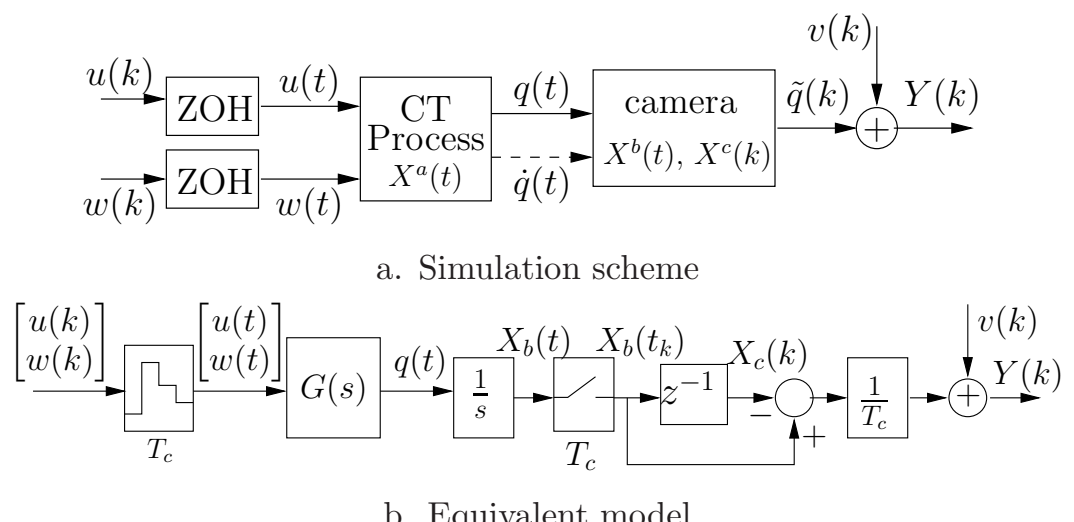

Figure 10: Block diagram of the hybrid model of the process with CBPM as sensor

The DT model of output $\tilde{q}$ and input $\left[\begin{array}{ll}u^{\mathrm{T}}(k) & w^{\mathrm{T}}(k)\end{array}\right]^{\mathrm{T}}$ writes $G(z)=$ $\frac{1}{T_{c}}\left(1-z^{-1}\right) \mathrm{c} 2 \mathrm{~d}\left(G_{a b}(s), T_{c},{ }^{\prime} z o h^{\prime}\right)$ where c2d $\left(\bullet, T_{c},{ }^{\prime} z o h^{\prime}\right)$ stands for the CT-toDT transform with the assumption of $\mathrm{ZOH}$ on input signals.

Looking more into the details, the resulting model, based on state vector $X(k)=\left[\begin{array}{c}X_{a}\left(t_{k}\right) \\ X_{b}\left(t_{k}\right) \\ X_{c}(k)\end{array}\right]$, has a SS representation of the form:

$$
\begin{aligned}
X(k+1) & =A X(k)+B_{1} u(k)+B_{2} w(k) \\
Y(k) & =C X(k)+v(k)
\end{aligned}
$$

Provided that matrices $A_{a a}, A_{a b}, A_{b a}, A_{b b}, B_{a u}, B_{a w}, B_{b u}$ and $B_{b w}$ represent a partition of the SS matrices of the DT model from $\left[\begin{array}{l}u(k) \\ w(k)\end{array}\right]$ to $X_{b}\left(t_{k}\right)$ with state vector $\left[\begin{array}{l}X_{a}\left(t_{k}\right) \\ X_{b}\left(t_{k}\right)\end{array}\right]$ obtained with $\mathrm{c} 2 \mathrm{~d}\left(G_{a b}\left(s, T_{c},{ }^{\prime} z o h^{\prime}\right)\right.$, the state matrices $A, B$, $C$ write $A=\left[\begin{array}{ccc}A_{a a} & A_{a b} & O_{n_{G} \times n_{q}} \\ A_{b a} & A_{b b} & O_{n_{q} \times n_{q}} \\ O_{n_{q} \times n_{G}} & I_{n_{q} \times n_{q}} & O_{n_{q} \times n_{q}}\end{array}\right], B_{1}=\left[\begin{array}{c}B_{a u} \\ B_{b u} \\ O_{n_{q} \times n_{u}}\end{array}\right], \quad B_{2}=\left[\begin{array}{c}B_{a w} \\ B_{b w} \\ O_{n_{q} \times n_{q}}\end{array}\right]$ and $C=\left[\begin{array}{lll}O_{n_{q} \times n_{G}} & \frac{1}{T_{c}} I_{n_{q} \times n_{q}} & \frac{-1}{T_{c}} I_{n_{q} \times n_{q}}\end{array}\right]$.

A pole-zero cancellation method (for instance minreal with Matlab) provides a reduced-order model based on state vector $\bar{X}$ such that $X=U \bar{X}$ and transforms the state matrices into the Kalman decomposition $\bar{A}=U^{-1} A U$, 
$\bar{B}=U^{-1} B, \bar{C}=C U$. This model $\{\bar{A}, \bar{B}, \bar{C}\}$ can be used for control and observation.

\section{Experimental evaluation}

\subsection{Evaluation method}

Experimental validation of the models requires to move a marker at high speed along a given trajectory in a reliable and reproducible way. A convenient setup has been developed for this purpose, based on a high-speed video projector [20] that projects a spot on a screen (see Fig. 11 and 12). A periodic trajectory is chosen and images are acquired by the camera at frame rate $f_{c}=1 / T_{c}$. Assuming that the projector rate $f_{p}$ is high, the displacement of the spot can be considered as continuous and the obtained images are equivalent to those obtained by the displacement of a LED on a moving arm. Assuming that the corresponding CT trajectory in the camera frame is known, the CBPM model will be used to predict the measurements. This prediction will be compared with the measurements based on image processing, thus allowing to evaluate the model.

Notice that the proposed setup has several advantages compared to a more conventional robotic system that would move the visual marker at different velocities. Indeed, the projection setup has no mechanical dynamics. Therefore, it is possible to move the target at arbitrary velocity with a very high accuracy in the marker position. Contrarily, the trajectory produced by a robotic setup would be altered by the dynamics at high frequency.

The steps required for validating the model are: (i) choose a trajectory in the projector frame; (ii) identify the corresponding trajectory in the camera frame; (iii) acquire the images for the corresponding trajectory; (iv) compute the prediction based on the model; (v) synchronize the data (in the case where no synchronization between camera and projector is available as herein); (vi) compare the output data. In the results presented herein, a circular trajectory was chosen.

\subsection{Description of the setup}

The experimental system, shown in Fig. 11 and 12, consists of a highspeed projector that generates marker patterns and a CCD camera that captures images of the marker. The projector and the camera are set approximately side by side at the distance of about $200 \mathrm{~mm}$ from each other. A 


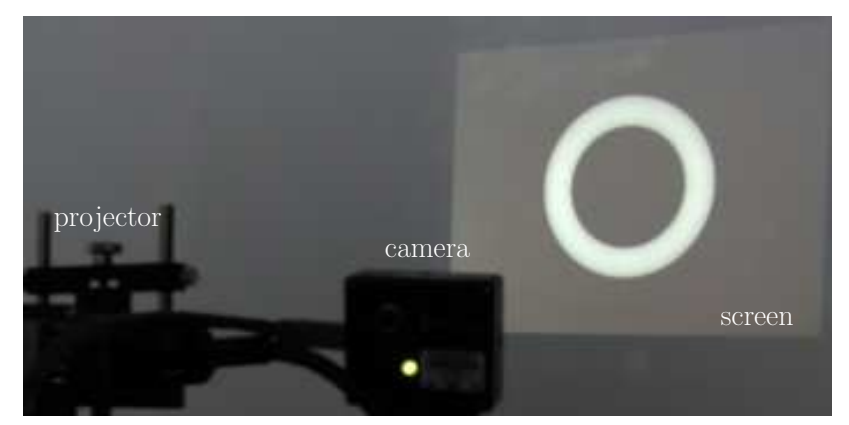

Figure 11: View of the setup; on the front-ground: the camera (right) and the videoprojector (left); on the background: the screen (N.B. the circle is obtained by the rotation of the marker over the exposure time of the camera)

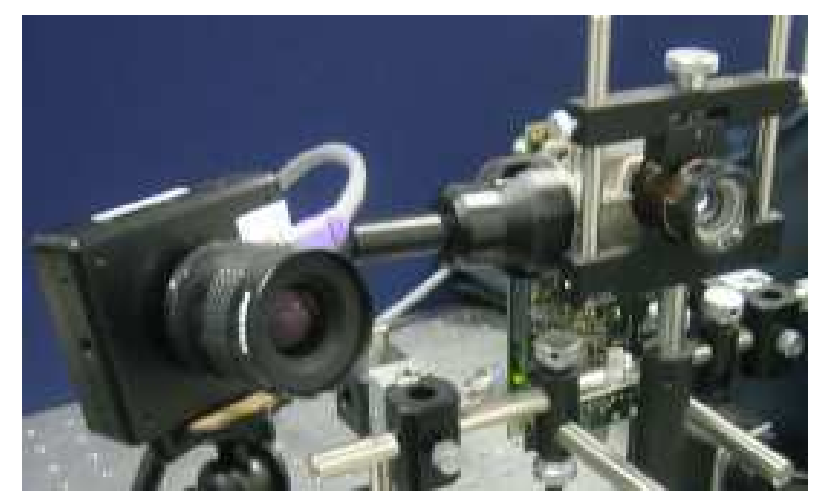

Figure 12: Partial view on the camera (left) and the videoprojector (right) 
planar screen is set at the distance of about $700 \mathrm{~mm}$ in front of the projectorcamera system.

The projector is composed of a Texas Instruments Digital Micromirror Device [21] DMD 0.7XGA 12 DDR, its controller board DMD Discovery 1100 and an external memory board ViALUX ALP-1. In this configuration, $1024 \times 768$ pixels binary images can be projected at up to $8 \mathrm{kHz}$. In the presented experiments, the maximum projection frame rate is set at $f_{p \max }=$ $4 \mathrm{kHz}$ to ensure stable operations. The external memory board is able to store up to $n_{p \max }=2730$ frames, and an arbitrary binary image sequence whose length is within $n_{p \text { max }}$ frames can be projected repeatedly. Light from a metal halide lamp Fiber-Lite MH-100 is guided to the DMD by an optical fiber guide, and the light reflected by the DMD is projected through a lens whose focal length is about $27 \mathrm{~mm}$. Since a single mirror size of the DMD is $13.68 \mu \mathrm{m}$, the horizontal view angle is about 29 degrees.

The camera is a Point Grey Research Dragonfly Express IEEE1394b camera, which captures $640 \times 480$-pixel 8-bit monochrome images with the progressive scan in GSM. In the presented experiments, images are acquired at $f_{c}=50 \mathrm{~Hz}$ with full-time exposure $\left(T_{e}=T_{c}\right)$. The focal length of the camera lens is about $6 \mathrm{~mm}$ and the CCD pixel size is $7.4 \mu \mathrm{m}$, resulting in an horizontal view angle of about 43 degrees.

For illustration, an image obtained with this system is given in Fig. 13.a, in addition with the measurements of the center of mass obtained with (Fig. 13.b) and without (Fig. 13.c) Sobel filter. Notice that the projector and the camera work in asynchronous mode.

\subsection{Trajectory design}

Consider a given periodic trajectory at frequency $f=1 / T$. It is necessary to choose the corresponding number of projector patterns $n_{p}$ and projector frame rate $f_{p}$ that satisfy the constraints $n_{p} \leq n_{p \max }$ and $f_{p} \leq f_{p \max }$. Let $T_{g}$ denote a common multiple of the different periods. Then, there exist integers $n_{p}, n$ and $n_{c}$ such that:

$$
T_{g}=n_{p} T_{p}=n T=n_{c} T_{c}
$$

where $T_{p}=1 / f_{p}$. In order to be close as possible to a CT trajectory, the projector rate $f_{p}$ is maximized.

Each projected image is composed of a white point of radius $r$ at a particular position on a circle of center $\left(x_{0}, y_{0}\right)$ and radius $R$. The trajectory in the 


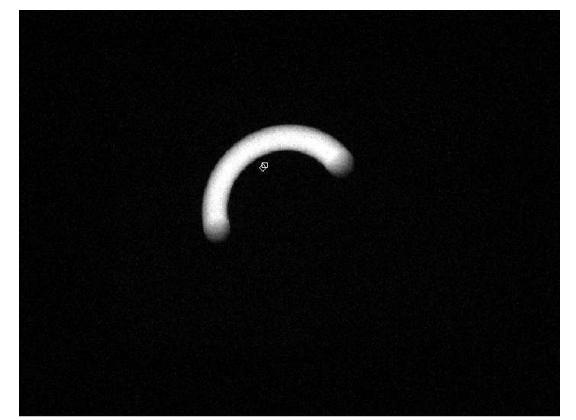

$a$.

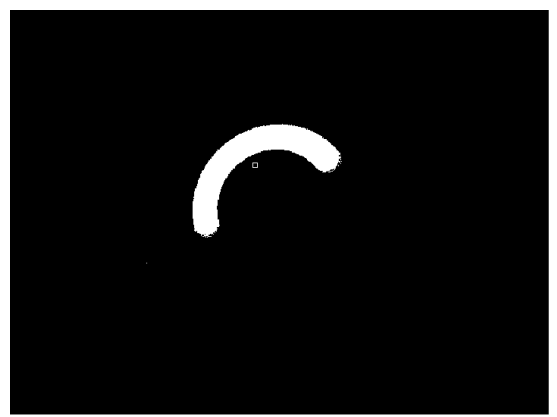

$b$.

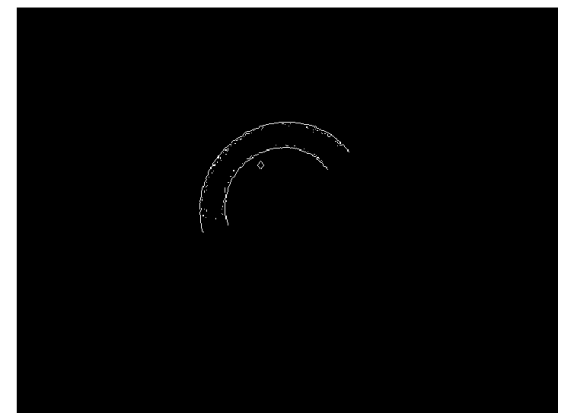

$c$.

Figure 13: Images obtained by the camera and image processing in addition with the position measurements. a/ original image and the measurements ( $\square$ : without Sobel filter; $\diamond$ : with Sobel filter); b/ binary image obtained without Sobel filter and its center of mass $(\square)$; c/ binary image obtained with Sobel filter and its center of mass $(\diamond)$

projector frame writes $x_{p}(t)=x_{0}+R \cos (\phi(t)), y_{p}(t)=x_{0}+R \sin (\phi(t))$ where $\phi(t)$ denotes the instantaneous phase. The center of the trajectory is chosen as the center of the image. The trajectory radius is chosen as $R=200$ pixels. For most of the tests, we chose $r=40$ pixels. However, experiments with different values of $r$ were processed. A number of $n_{p}$ frames are computed and projected at the projector frequency, based on $\phi_{k}=\phi\left(k T_{p}\right), k=0, \ldots, n_{p}-1$.

\subsection{Identification of the trajectory in the camera frame}

In order to validate the camera models, a projective model is necessary to provide the position in the camera frame for a given position in the projector frame. Taking the advantage of the periodicity of the movement, a simple approach is used consisting in a truncated Fourier series of the trajectory rather than a real calibration.

Let $\left(x_{p}(\phi), y_{p}(\phi)\right)$ denotes the known trajectory in the projector frame and $(x(\phi), y(\phi))$ the unknown trajectory in the image frame, with the same periodicity $2 \pi$. The following truncated Fourier series can be used:

$$
\begin{aligned}
& x(\phi)=a_{0}+\sum_{k=1}^{m} a_{k} \cos (k \phi)+b_{k} \sin (k \phi) \\
& y(\phi)=c_{0}+\sum_{k=1}^{m} c_{k} \cos (k \phi)+d_{k} \sin (k \phi)
\end{aligned}
$$

Parameters $a_{k}, b_{k}, c_{k}$ and $d_{k}$ are evaluated by linear regression in order to fit static measurements obtained for several values of $\phi$ in $[0 ; 2 \pi]$. A number 


\begin{tabular}{cccccc}
$\lambda^{*}$ & $n$ & $n_{c}$ & $\lambda=\frac{n}{n_{c}}$ & $T_{g}(\mathrm{~ms})$ & $n_{p}$ \\
\hline 0.1 & 1 & 10 & 0.1 & 200 & 800 \\
0.192 & 1 & 8 & 0.125 & 160 & 640 \\
0.167 & 1 & 6 & 0.167 & 120 & 480 \\
0.215 & 1 & 5 & 0.2 & 100 & 400 \\
0.278 & 3 & 11 & 0.272 & 220 & 880 \\
0.359 & 1 & 3 & 0.333 & 60 & 240 \\
0.464 & 6 & 13 & 0.462 & 260 & 1040 \\
0.599 & 3 & 5 & 0.6 & 100 & 400 \\
0.774 & 3 & 4 & 0.75 & 80 & 320 \\
1 & 1 & 1 & 1 & 20 & 80
\end{tabular}

Table 3: Numerical values of the parameters of the constant velocity experiment; $T_{g}$ is the multiple period and $n_{p}$ is the number of frames.

of 1024 positions regularly spaced was chosen. Order $m=2$ was chosen for the truncation, allowing an accuracy around 0.13 pixel, which is close to the residual measurement error.

\subsection{Evaluation results}

\subsubsection{Constant velocity trajectories}

The phase of the trajectory writes $\phi(t)=2 \pi f t$. Several experiments were proceeded with various $\lambda=f / f_{c}=n / n_{c}$, i.e. various ration of the trajectory frequency to camera frame rate, from 0.1 to 1 . Making use of the explanations of Section 3.2, $n$ and $n_{c}$ were chosen manually such that $n / n_{c}$ is close to the desired value $\lambda^{*}$ of $\lambda$. The maximum projector rate $f_{p}=4 \mathrm{kHz}$ was used for each case. The parameters obtained for 10 values of $\lambda^{*}$ logarithmically spaced are given in Table 3.

As an example, the results for $\lambda=0.333$ are given in Fig. 14. One can notice that model $\mathcal{M}_{2}$ allows very accurate predictions of the measurements. Remind that $\mathcal{M}_{1}$ and $\mathcal{M}_{2}$ provide the same outputs for constantvelocity trajectories. For evaluation of the models, the mean distance error $J=\frac{1}{m} \sum_{k=1}^{m} \sqrt{\left(\Delta x_{m}(k)\right)^{2}+\left(\Delta y_{m}(k)\right)^{2}}$ is considered in the sequel, where $\Delta x_{m}(k)$ and $\Delta y_{m}(k)$ are respectively the errors on $x$ and $y$ coordinates for measurement $\# k, k=1, \ldots, m$, . The evaluations obtained for the different values of the ratio $f / f_{c}$ are given in Fig. 15 considering 5 different models $\mathcal{M}_{2}$ to $\mathcal{M}_{6}$ (see Tab. 2 for the list of the models). One can notice that model 

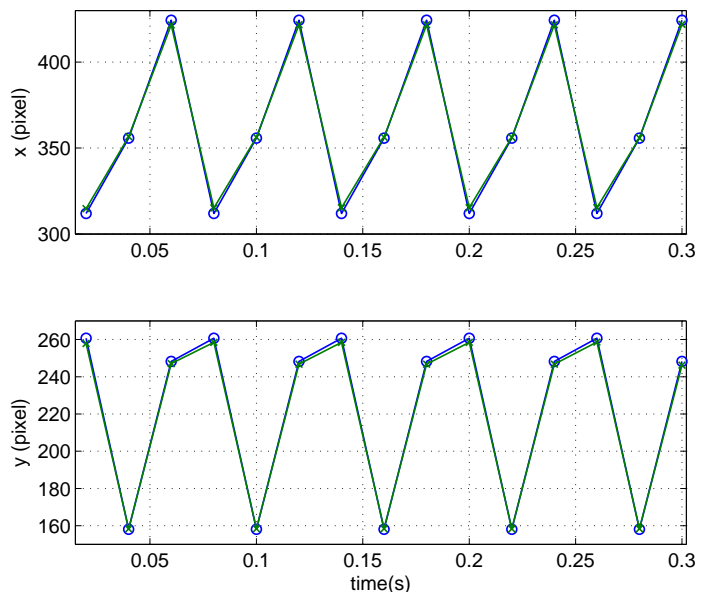

Figure 14: Measurements $(+)$ and predictions with model $\mathcal{M}_{2}(\circ)$ for $\lambda=0.359$

$\mathcal{M}_{2}$ developed in this paper is by far the more accurate. This is notably the case when the signal frequency is close to the camera rate. Whereas the mean distance error $J$ remains lower than 2 pixels with the proposed model, it reaches or exceeds 9 pixels with the other models. One can also notice that models $\mathcal{M}_{5}$ and $\mathcal{M}_{6}$ are efficient for low ratios of trajectory to camera frequencies, but the prediction error increases for frequencies close to the camera rate.

\subsubsection{Trajectories with varying velocity}

Models $\mathcal{M}_{1}$ and $\mathcal{M}_{2}$ coincide for constant-speed trajectories $\left(\mathcal{H}_{4}\right.$ is the only assumption that is assumed by $\mathcal{M}_{2}$ and not by $\mathcal{M}_{1}$ ), which was the case for constant phase velocity $\dot{\phi}$ in the previous experimental setup. In order to emphasize the better accuracy of model $\mathcal{M}_{1}$ for variable speed, an experiment was done where the angular speed $\dot{\phi}$ of the trajectory varies from approximately $100 \mathrm{rad} / \mathrm{s}$ to $300 \mathrm{rad} / \mathrm{s}$ with a periodicity of approximately $50 \mathrm{~ms}$. The numerical values were chosen such that all the frequencies have a common periodicity. A portion of the chosen trajectory is given in Fig. 16. One can notice that after 11 camera periods and 7 turns, the marker comes back to the initial position. Over a camera period, the shift in angle $\phi$ varies from 2.78 to $5.29 \mathrm{rad}$. This extreme situation (compared to usual situations where the displacement of the marker over a camera period is small) is intended to discriminate the different models. 


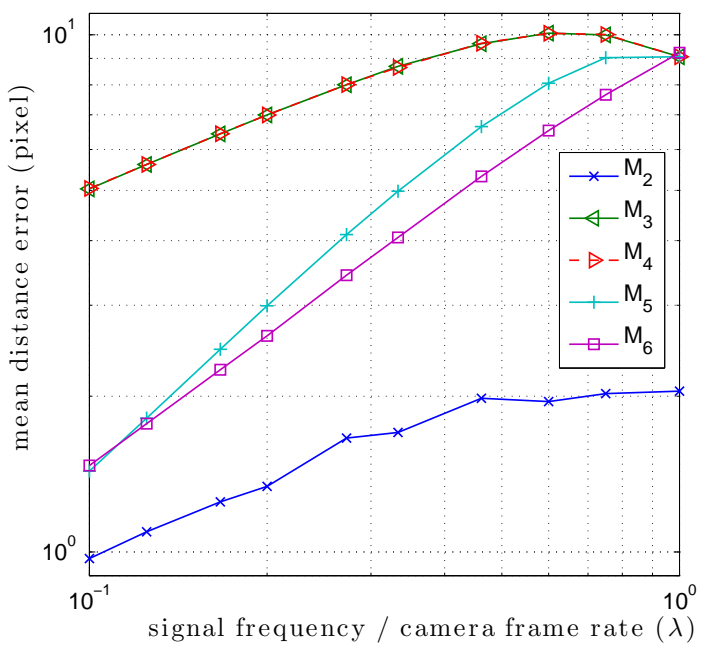

Figure 15: Variations with respect to $\lambda=f / f_{c}$ of the errors in the prediction obtained with the different models
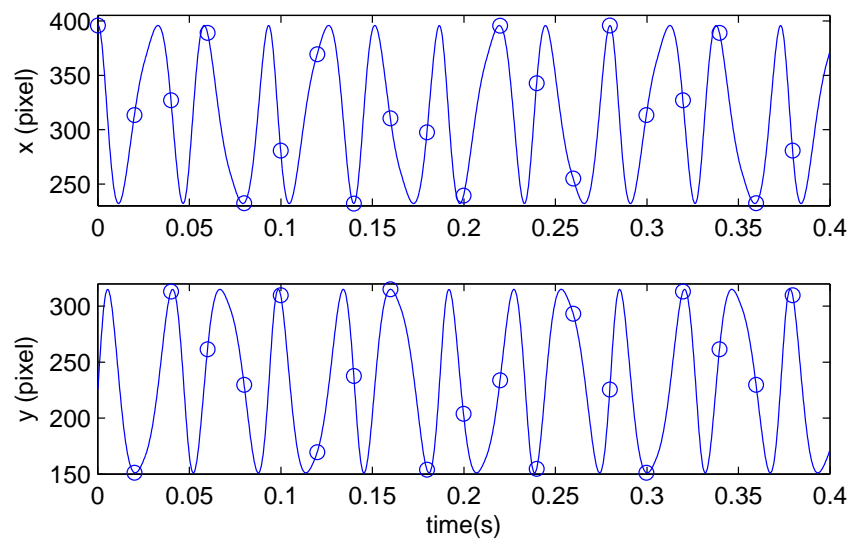

Figure 16: Variable speed circular trajectory for model evaluation (the circles represent the values sampled at the camera rate) 

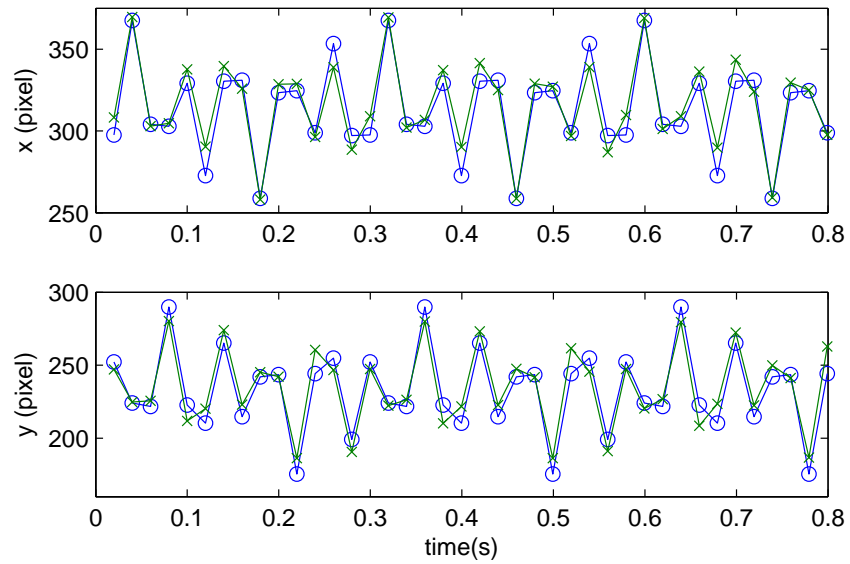

Figure 17: Comparison between measurement $(\times)$ and results provided by model $\mathcal{M}_{1}$ (o)

The values of the criterion $J$ (i.e. the mean distance error) for each model are given in Table 4 . One can notice that models $\mathcal{M}_{1}$ and $\mathcal{M}_{2}$ provide more accurate results than the other models. Model $\mathcal{M}_{1}$ is sensibly superior to $\mathcal{M}_{2}$ : the mean error is reduced by $25 \%$. For illustration, the prediction of the measurements with model $\mathcal{M}_{1}$ are given in Fig. 17 and compared with the values obtained experimentally. One can notice that the model reproduces the measurements with rather good precision.

\begin{tabular}{|c||c|c|c|c|c|c|}
\hline model & $\mathcal{M}_{1}$ & $\mathcal{M}_{2}$ & $\mathcal{M}_{3}$ & $\mathcal{M}_{4}$ & $\mathcal{M}_{5}$ & $\mathcal{M}_{6}$ \\
\hline$J$ (pixel) & 12.6 & 16.9 & 94.3 & 100.7 & 66.3 & 86.1 \\
\hline
\end{tabular}

Table 4: Mean distance error between measurements and the models

\subsubsection{Varying the spot radius and the detection algorithm}

Due to Assumption $\mathcal{H}_{1}$, the radius of the spot is assumed to be small compared to the radius of the trajectory. It is then interesting to check the effect of the marker size on the accuracy of the model. The experiments were made at constant velocity with markers of different radii $r$. The results obtained with $k_{1}=3$ and $k_{2}=5$ are given with two processing protocols for image processing:

- with threshold and center of mass, Fig. 18, 


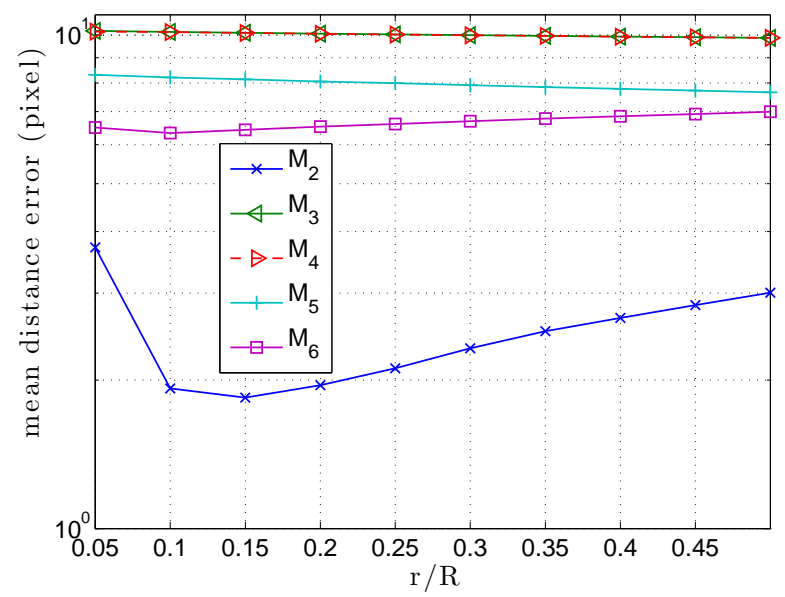

Figure 18: Influence of the marker radius without Sobel filter

- with Sobel filter, threshold and center of mass, Fig. 19.

One can notice that the estimation error varies with respect to $r / R$. Let focus on Model $\mathcal{M}_{2}$. As expected, the error globally increases with $r / R$. In the case without Sobel filter, the mean distance error $J$ is higher for the very low $r / R(r / R \leq 0.1)$. Indeed, in this situation, images receive few light and the detection of the center of mass is hazardous. Assuming that the marker is sufficiently luminous, model $\mathcal{M}_{2}$ is sensibly more accurate without using the Sobel filter.

\section{Compensation of the dynamic effects using a Kalman filter}

\subsection{Presentation of the issue}

It is not possible to invert the camera model, i.e. to determine the CT trajectory $q(t)$ only from the DT measurement $q_{m}(k)$. Therefore, additional information must be used. When a model of the process generating $q(t)$ is available, the Kalman filter is the most common tool allowing to combine the model and the output measurement in order to estimate the trajectory of the internal states. In this section, the use of the Kalman filter is explained for the particular issue of determining the CT trajectory of a marker position measured by camera. Whereas the methodology is rather general, the specific case of a camera with GSM and full exposure time is considered herein. 


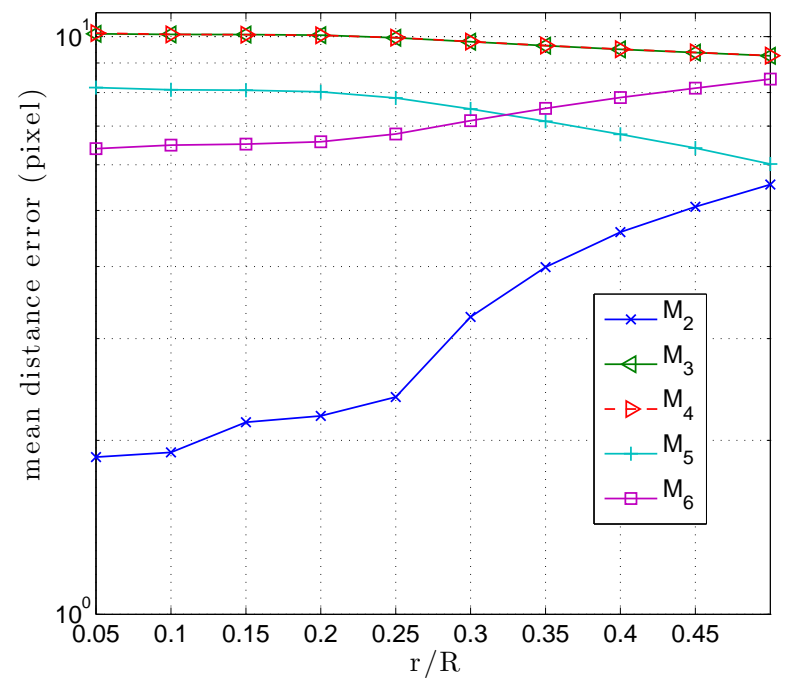

Figure 19: Influence of the marker radius with Sobel filter

The global model has the form shown in the diagram of Fig. 10. Input vector $u(k)$ corresponds to the known input signals (such as the control signals) and $w(k)$ corresponds to the noise that affects the CT process (unknown input signals are considered as noise). Without any other information, it is sensible to consider that the continuous signals $u(t)$ and $w(t)$ are constant over a sampling interval, i.e. they are provided through $\mathrm{ZOH}$. The process is $\mathrm{CT}$ and could be any kind of CT system. The measurement given by the camera is altered by some additive measurement noise $v(k)$. The dashed line with $\dot{q}(t)$ corresponds to the fact that $\dot{q}(t)$ would be necessary for model $\mathcal{M}_{1}$ but not for the other models. The state of the CT process is denoted by $X_{a}(t)$. In this paper, we consider that the CT process is linear and the linear model $\mathcal{M}_{2}$ of the CBPM is used. Extension to nonlinear systems is possible. Capital letters are used for the states $(X)$ and outputs $(Y)$ in order to distinguish them from the $x$ and $y$ coordinates.

\subsection{Kalman filter for discrete-time systems}

The Kalman filter allows to determine the estimate of the state with minimal variance [22]. Details on the theory and application of the Kalman filter can be found in many publications [23, 24]. Herein, a simplified version is given, corresponding to the case where the gain matrix $K$ has converged, 
resulting in a linear filter. At time $t_{k}$, the estimation is computed in two steps:

1. compute the prediction $\hat{\bar{X}}(k+1 \mid k)$ of the state at time $t_{k+1}$ from data available at $t_{k}$ :

$$
\hat{\bar{X}}(k+1 \mid k)=\bar{A} \hat{\bar{X}}(k \mid k)+\bar{B}_{1} u(k)
$$

2. compute the correction $\hat{\bar{X}}(k+1 \mid k+1)$ based on measurements at $t_{k+1}$ :

$$
\begin{aligned}
\hat{\bar{X}}(k+1 \mid k+1)= & \hat{\bar{X}}(k+1 \mid k)+K(Y(k+1) \\
& -\bar{C} \hat{\bar{X}}(k+1 \mid k)
\end{aligned}
$$

The gain matrix $K$ is computed using the observable model $\{\bar{A}, \bar{B}, \bar{C}\}$ developed in Subsection 2.5.

\subsection{Adaptation to the hybrid model}

The previous DT Kalman filter can be adapted for estimation of the CT trajectory $q(t)$ and the CT state of the process $X_{a}(t)$. First, the estimation scheme based on the reduced state $\bar{X}(k)$ can be transposed to $X(k)$, provided that the system is detectable. The corresponding estimation scheme is simply obtained from (24-25) by removing all the bars (i.e. using the state matrices of the model before pole-zero cancellation) and replacing the correction gain $K$ by $U K$.

Second, the CT nature of the signals to observe must be recovered. This is solved by replacing the computation of the prediction step (24) by:

1. simulation of the CT part of the model (states $X_{a}(t)$ and $X_{b}(t)$ ) on $\left[t_{k} ; t_{k+1}\right]$ by any numerical solver dedicated to ordinary differential equations,

2. computation of the DT part $\left(X_{c}(k+1)=X_{b}\left(t_{k}\right)\right)$.

The correction step, based on the DT measurements available at each camera period, is unchanged.

The resulting observer can be considered as a hybrid system in two ways: $1 /$ it contains both CT and DT states; 2 / the CT states make a jump at each sampling time, due to the correction step. Practically, the simulation of the CT part is done numerically at an arbitrarily small time-step. 


\section{Trajectory reconstruction}

We propose to show the interest of the methodology presented in Section IV by a specific application: the reconstruction of a periodic displacement of one marker.

\subsection{Experiment}

In heart-beating surgery, the portion of interest of the heart is immobilized by a mechanical stabilizer. However, some residual motion remains that act as a disturbance for the surgeon [11]. Several solutions have been proposed to improve the stability of the region of interest $[18,25,26]$. In order to obtain the best results, there is a need to predict the movement of the heart [27]. This can be done using LEDs attached on the tip of the stabilizer and measured by a camera. The estimation can be done by Kalman filter $[28]$.

The experimental setup shown in Fig. 1 has been used to reproduce the displacements of a beating heart. It consists of a pan-tilt arm equipped with seven LEDs at its tip. A fixed camera (DALSA CAD6) allows acquisition of images $(256 \times 256$ pixels $)$ at variable frequencies, up to $400 \mathrm{~Hz}$. The pantilt arm is controlled in position at $500 \mathrm{~Hz}$. The chosen position reference corresponds to a periodic movement corresponding to one cardiac period of the heart movement recorded by Ginhoux et al. [29]. The corresponding trajectory in the image is given in plain line in Fig. 20.

The goal of the study is to show that the use of the camera model allows to reconstruct the trajectory of a complex periodic movement with a low frequency camera whereas without the use of the model, a high-speed camera is required. Therefore, the movement of the platform is first acquired at a relatively high frequency to get an accurate knowledge of the real trajectory. A camera rate $f_{c 1}=250 \mathrm{~Hz}$ was considered as sufficient. The position of a LED is obtained as following: an area of interest centered on the previous position allows to discriminate one LED from the others; a Sobel filter detects the contour; the computation of the center of mass of the pixels of the contour is considered as the marker position. Trajectories at lower frequencies should be acquired for comparison. However, in order to compare synchronized trajectories, it was decided to reconstruct the images at lower frequencies. For instance, the trajectory at $f_{c 2}=25 \mathrm{~Hz}$ is obtained by superimposing $f_{c 1} / f_{c 2}=10$ consecutive images. As an illustration, the image computed form 10 successive images acquired at $250 \mathrm{~Hz}$ is shown in Fig. 21. One can 

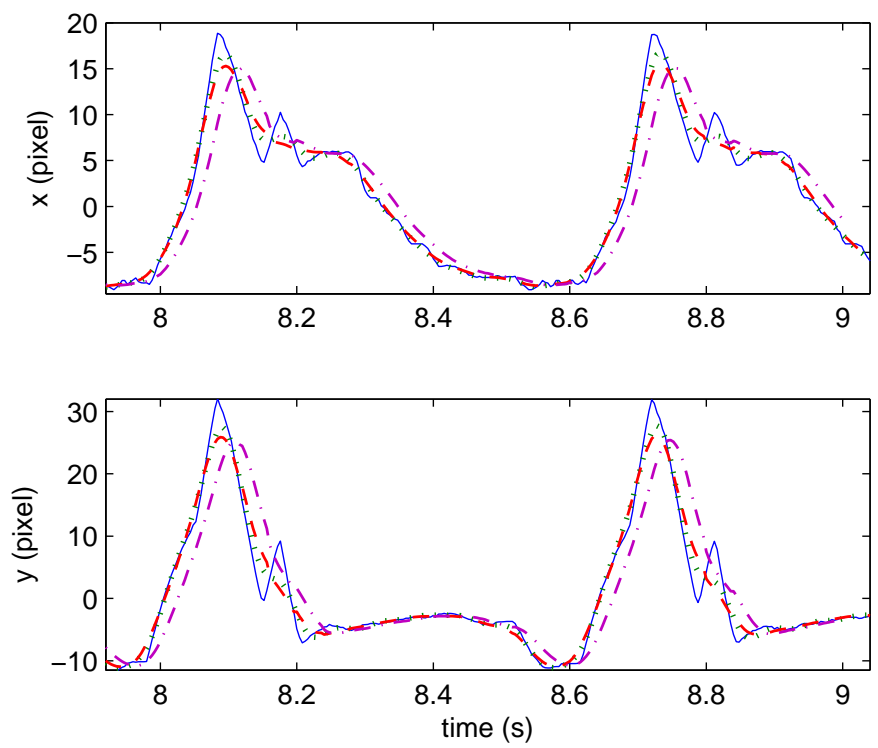

Figure 20: CT trajectory obtained in different conditions: $\mathcal{C}_{1}$ (measured at $250 \mathrm{~Hz}$, plain line), $\mathcal{C}_{2}$ (Fourier serial of order 9 estimated from measurements at $250 \mathrm{~Hz}$ without camera model, dots), $\mathcal{C}_{3}$ (Fourier serial of order 9 estimated with the linear model of the camera from images acquired at $25 \mathrm{~Hz}$, dashed line), $\mathcal{C}_{4}$ (Fourier serial of order 9 estimated at $25 \mathrm{~Hz}$ without camera model, dashed-dotted line).

see the resemblance with the image given in the right-hand side of Fig. 2 that was acquired at $25 \mathrm{~Hz}$.

\subsection{Signal model}

Let us explain how to model a periodic trajectory into the scheme of Fig. 10.a in order to use the Kalman filter with the methodology introduced in the previous section.

A periodic signal $x(t)$ of period $T$ and of finite bandwidth can be decomposed into a finite sum of harmonics $x_{i}(t), i=1, \ldots, n_{h}$, at multiple frequencies if where $f=1 / T$. Each harmonic can be produced by a second order resonant filter of equation $\ddot{x}_{i}(t)+\left(i \omega_{0}\right)^{2} x_{i}(t)=0$ of eigen-frequency $i \omega_{0}$ with $\omega_{0}=2 \pi f$. To allow the Kalman filter to converge to the right phase and amplitude, a noise $w_{i}^{x}(t)$ is considered on the acceleration, leading to $\ddot{x}_{i}(t)+\left(i \omega_{0}\right)^{2} x_{i}(t)=w_{i}^{x}(t)$. For the $y$ coordinate, the same approach is used; the corresponding second order filter has independent states $\left(y_{i}(t)\right.$ and $\left.\dot{y}_{i}(t)\right)$ and inputs $w_{i}^{y}(t)$. 


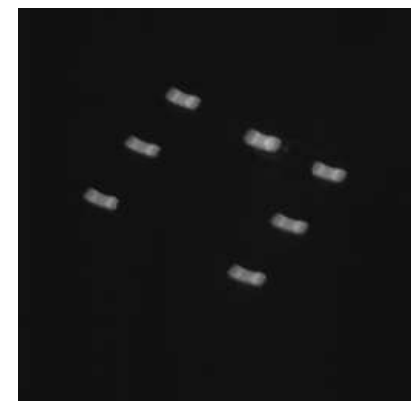

Figure 21: Image computed from 10 images acquired at $250 \mathrm{~Hz}$ for simulation of the $25 \mathrm{~Hz}$ acquisition.

By stacking all the $2^{\text {nd }}$ order blocks, a model of the process can be obtained $^{3}$. Its state $X_{a}$ is of order $4 n_{h}$. The input vector $w$ is of order $2 n_{h}$. The outputs $x(t), y(t), \dot{x}(t)$ and $\dot{y}(t)$ are respectively computed as the sum of the different harmonics $x_{i}(t), y_{i}(t), \dot{x}_{i}(t)$ and $\dot{y}_{i}(t)$. This model is linear and has no direct feed-through; it has no control input $u$.

\subsection{Filter tuning}

A correct tuning of the covariance matrices is necessary in order to obtain a good behavior of the Kalman filter. The noise $v(k)$ on measurement generally satisfies the assumptions of time-independence and zero mean. Therefore, its covariance matrix $R$ can be tuned according to an estimation of the noise amplitude. In our case, the standard deviation of the measurement is about 0.1 pixel, leading to $R=0.01 \times \mathbb{I}_{2}$ where $\mathbb{I}_{2}$ is the identity matrix of order 2 .

The tuning of matrix $Q$ is a more difficult question as input $w(k)$ is an artifact used for allowing the filter to adapt to the real trajectory. It is sensible to restrict it to a diagonal matrix with the same values for the $x$ and $y$ coor-

${ }^{3}$ For the harmonic of rank $\# i$, the model writes

$$
\left[\begin{array}{c}
\dot{x}_{i} \\
\ddot{x}_{i} \\
\dot{y}_{i} \\
\ddot{y}_{i}
\end{array}\right]=\left[\begin{array}{cccc}
0 & 1 & 0 & 0 \\
-i^{2} \omega_{0}^{2} & 0 & 0 & 0 \\
0 & 0 & 0 & 1 \\
0 & 0 & -i^{2} \omega_{0}^{2} & 0
\end{array}\right]\left[\begin{array}{c}
x_{i} \\
\dot{x}_{i} \\
y_{i} \\
\dot{y}_{i}
\end{array}\right]+\left[\begin{array}{cc}
0 & 0 \\
1 & 0 \\
0 & 0 \\
0 & 1
\end{array}\right]\left[\begin{array}{c}
w_{i}^{x} \\
w_{i}^{y}
\end{array}\right]
$$

The state matrices of the complete model of $G(s)$ are obtained by stacking the matrices obtained for $i=1, \ldots, n_{h}$ in a block-diagonal fashion. 
dinates of a given harmonic, yielding $Q=\operatorname{diag}\left(Q_{1}, Q_{1}, Q_{2}, Q_{2}, \ldots, Q_{n_{h}}, Q_{n_{h}}\right)$. There remains the tuning of $n_{h}$ positive scalars. Notice that a higher value of $Q_{j}$ corresponds to more noise on $w_{j}^{x}$ and $w_{j}^{y}$ and will allow the filter to adapt faster. However, a fast adaptation results in high sensitivity to the measurement noise. A trade-off needs to be found. As the amplitude of the harmonics generally decrease with the frequency, we chose $Q_{j}$ to decrease hyperbolically in $j$, i.e. $Q_{j}=Q_{1} / j, j=2, \ldots, n_{h}$. When the number $n_{h}$ of considered harmonics vary, there is a need to adapt the tuning. Indeed, a higher $n_{h}$ corresponds to more components in $w$ and then more possibility for the filter to adapt. Therefore, we decided to choose $Q_{1}=Q_{0} / n_{h}$ for reducing the weight with respect to the number of harmonics. Finally, the value of $Q_{0}=100$ was chosen so that the filter converges after a few periods of the signal.

\subsection{Results}

In order to evaluate the interest of including the camera model in the estimation model, the trajectory was estimated at different frequencies with or without camera model and compared. Different curves in the conditions mentioned in Tab. 5 are given in Fig. 20. $\mathcal{C}_{1}$ is the trajectory obtained at $250 \mathrm{~Hz}$. This trajectory is considered to be the actual trajectory of $(x(t), y(t))$. The other trajectories are reconstructed by Kalman filter with $n_{h}=9$ harmonics in different conditions: in $\mathcal{C}_{2}$, images are acquired at $250 \mathrm{~Hz}$. Notice that at this frequency, the camera does not have much effect, therefore, this trajectory is the reference for the reconstruction of the truncated signal. The other curves $\left(\mathcal{C}_{3}\right.$ to $\left.\mathcal{C}_{6}\right)$ are obtained from images acquired at $25 \mathrm{~Hz}$. $\mathcal{C}_{3}$ is obtained with the linear camera model $\mathcal{M}_{2}, \mathcal{C}_{4}$ without any camera model and $\mathcal{C}_{5}$ with camera model $\mathcal{M}_{6}$ (it corresponds to trajectory $\mathcal{C}_{4}$ time-shifted of $\left.T_{c} / 2\right)$.

\begin{tabular}{|c||c|c|c|c|c|}
\hline curve & $\mathcal{C}_{1}$ & $\mathcal{C}_{2}$ & $\mathcal{C}_{3}$ & $\mathcal{C}_{4}$ & $\mathcal{C}_{5}$ \\
\hline camera rate $(\mathrm{Hz})$ & 250 & 250 & 25 & 25 & 25 \\
\hline camera model & $/$ & $/$ & $\mathcal{M}_{2}$ & $/$ & $\mathcal{M}_{6}$ \\
\hline order $n_{h}$ & $\infty$ & 9 & 9 & 9 & 9 \\
\hline
\end{tabular}

Table 5: The different curves considered for evaluation 

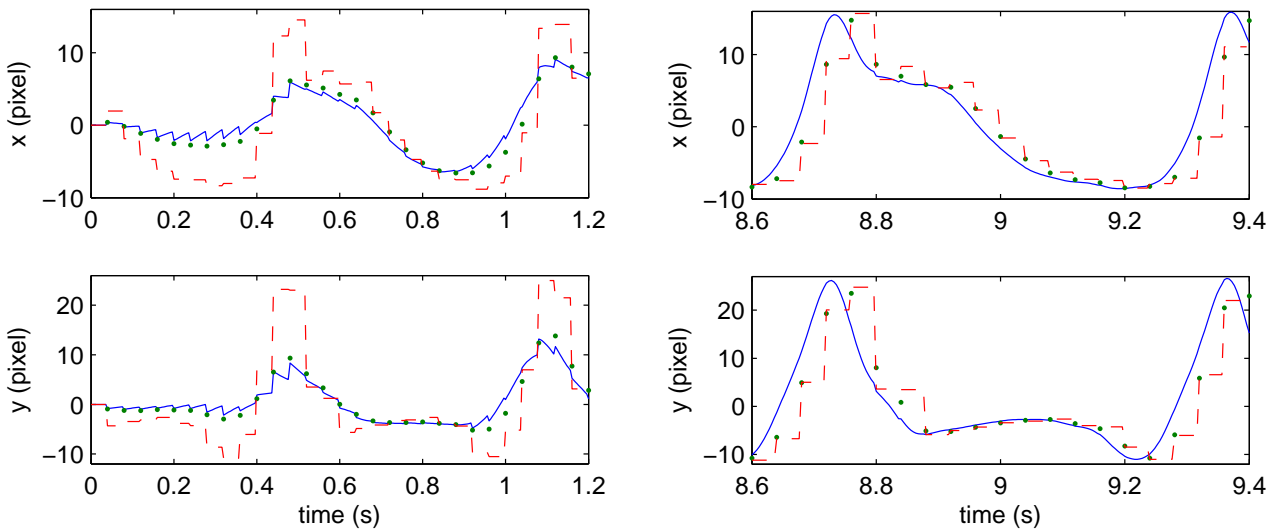

Figure 22: Estimation results with the linear model of the camera $\left(\mathcal{M}_{2}\right)$. The estimated CT trajectory is given in plain line. The blocked signal in dashed line corresponds to the measurement at $25 \mathrm{~Hz}$. The corrected estimation of the measurement is given in dot. On the left is presented the beginning of the estimation; the estimated curve seems to be attracted by the measurements. On the right is presented the behavior after convergence; the measurements are accurately estimated (the points belong to the dashed curve).

As an illustration of the behavior of the hybrid Kalman filter, the results $\left(\mathcal{C}_{3}\right)$ obtained with the linear model $\left(\mathcal{M}_{2}\right)$ at $25 \mathrm{~Hz}$ are detailed in Fig. 22. During the convergence phase (left-hand side), one can notice the jumps in the CT trajectory at the measurement instants. After convergence (plot on the right-hand side), the estimated measurements (dots) are very close to the effective measurements (dashed line) and the discontinuities due to the correction have vanished.

The different curves being sampled with the same frequency $f_{c 1}=250 \mathrm{~Hz}$, the mean distance between two curves, after convergence, is given in Tab. 6 for several couples of curves. The comparisons are given with respect to the actual trajectory $\mathcal{C}_{1}$ and with respect to the estimation from the Kalman filter at high frequency, i.e. $\mathcal{C}_{2}$. Remind that Curves $\mathcal{C}_{1}$ to $\mathcal{C}_{4}$ are shown in Fig. 20.

Let $d\left(\mathcal{C}_{k}, \mathcal{C}_{l}\right)$ denote the mean distance between $\mathcal{C}_{k}$ and $\mathcal{C}_{l}$ reported in Tab. 6. Let us start with comparisons with respect to $\mathcal{C}_{1}$. The value $d\left(\mathcal{C}_{1}, \mathcal{C}_{2}\right)=1.46$ evaluates the approximation due to the Fourier series truncation at order 9 . Notice that this error can be reduced by increasing the order $n_{h}$ of the Fourier series. When estimating the trajectory with a lowfrequency camera $\left(\mathcal{C}_{4}\right)$, it appears that this error nearly triples if no camera 


\begin{tabular}{|c||c|c|c|c|}
\hline$d\left(\mathcal{C}_{k}, \mathcal{C}_{l}\right)$ & $\mathcal{C}_{2}$ & $\mathcal{C}_{3}$ & $\mathcal{C}_{4}$ & $\mathcal{C}_{5}$ \\
\hline $\mathcal{C}_{1}$ & 1.46 & 1.76 & 4.24 & 3.13 \\
\hline $\mathcal{C}_{2}$ & 0 & 0.60 & 3.00 & 2.33 \\
\hline
\end{tabular}

Table 6: Comparison of the different curves: mean distance $d$ (in pixel)

model is used (compare $d\left(\mathcal{C}_{1}, \mathcal{C}_{4}\right)$ with $\left.d\left(\mathcal{C}_{1}, \mathcal{C}_{2}\right)\right)$ but, with the camera model $\mathcal{M}_{2}$ (curve $\left.\mathcal{C}_{3}\right)$ ), the error is brought back to a similar value (see $d\left(\mathcal{C}_{1}, \mathcal{C}_{3}\right)$ ).

Now, let us make comparisons with respect to $\mathcal{C}_{2}$, considered as the reference for the estimation of a truncated signal. The use of the camera model $\mathcal{M}_{2}$ in $\mathcal{C}_{3}$ highly improves the results compared to those obtained in $\mathcal{C}_{4}$ without camera model, the distance being divided by 5 (see $d\left(\mathcal{C}_{4}, \mathcal{C}_{2}\right)$ compared to $\left.d\left(\mathcal{C}_{3}, \mathcal{C}_{2}\right)\right)$. As shown with the results obtained with curve $\mathcal{C}_{6}$, model $\mathcal{M}_{5}$ is not as efficient as model $\mathcal{M}_{2}$ (see $d\left(\mathcal{C}_{5}, \mathcal{C}_{2}\right)$ compared to $d\left(\mathcal{C}_{3}, \mathcal{C}_{2}\right)$ ). On can observe that the camera model allows to obtain results with a $25 \mathrm{~Hz}$ camera that are very similar to those obtained at $250 \mathrm{~Hz}$ without camera model.

\section{Conclusion}

This paper proposes an insight visit of the dynamic behavior of CBPM. Different models have been developed, depending on the camera technology (GSM or RSM) for full or partial exposure time. A nonlinear model $\mathcal{M}_{1}$ was first developed. It allows to reproduce the measurement with better accuracy than all the other models. Therefore, it is suitable for refined simulation. For estimation and control, the linear model $\mathcal{M}_{2}$, assuming constant velocity over the exposure time, is more suitable. An experimental setup and a complete procedure have been proposed for the model validation. The results showed the significant improvements obtained with these models compared to the reference models. Notice that the paper focuses on the $2 \mathrm{D}$ movement of points in the image. Investigations of the dynamic effects of other visual features such as lines, and also the effects on a reconstructed 3D trajectory remains to be considered.

In addition, this paper presents a methodology to account for the dynamic effects of the camera when reconstructing the trajectory of a visual marker. Making use of a model of the camera, a Kalman filter is used to estimate the CT trajectory. The methodology was evaluated in the context of the recon- 
struction of an unknown periodic signal, corresponding to the displacements of a beating heart. In this context, it was shown that the use of the model allows to obtain results with a low frame-rate camera that resemble those obtained with a high-speed camera. For instance, the trajectory estimated with a Fourier series could be estimated at $25 \mathrm{~Hz}$ with an accuracy close to what is given by a camera at $250 \mathrm{~Hz}$ without camera model.

\section{References}

[1] P. Corke, Visual Control of Robots, Research Studies Press Ltd., Taunton, Somerset, U.K., 1996.

[2] R. Andersson, Dynamic sensing in a ping-pong playing robot, IEEE Transactions on Robotics and Automation 5 (6) (1989) 728 - 739.

[3] Y. Nakabo, M. Ishikawa, H. Toyoda, S. Mizuno, 1 ms column parallel vision system and its application of high speed target tracking, in: International Conference on Robotics and Automation, Vol. 1, 2000, pp. $650-655$.

[4] H. Chen, K. Matsumoto, J. Ota, T. Arai, Self-calibration of environmental camera for mobile robot navigation, Robotics and Autonomous Systems 55 (3) (2007) 177 - 190.

[5] G. Klein, T. Drummond, A single-frame visual gyroscope, in: Proc. British Machine Vision Conference, Vol. 2, Oxford, 2005, pp. 529-538.

[6] G. Boracchi, Estimating the 3D direction of a translating camera from a single motion-blurred image, Pattern Recognition Letters 30 (2009) 671-681.

[7] C. Liang, L. Chang, H. Chen, Analysis and compensation of rolling shutter effect, IEEE Transactions on Image Processing 17 (8) (2008) 1323-1330.

[8] O. Ait-Aider, A. Bartoli, N. Andreff, Kinematics from lines in a single rolling shutter image, in: IEEE Conference on Computer Vision and Pattern Recognition, Minneapolis, Minnesota, USA, 2007. 
[9] A. Ranftl, L. Cuvillon, J. Gangloff, J. van der Sloten, High speed visual servoing with ultrasonic motors, in: International Conference on Robotics and Automation, 2007.

[10] C. Luna, M. Mazo, J. Lazaro, J. Vazquez, J. Urena, S. Palazuelos, J. Garcia, F. Espinoza, E. Santiso, Method to measure the rotation angles in vibrating systems, IEEE Transactions on Instrumentation and Measurement 55 (1) (2006) 232-239.

[11] L. Cuvillon, J. Gangloff, M. de Mathelin, A. Forgione, Towards robotized beating heart tecabg: assessment of the heart dynamics using high-speed vision, Computer Aided Surgery 11 (5) (2006) 267-277.

[12] E. Laroche, J. Delavigne, Dynamical effects of vision-based position measurement, in: IFAC World Congress, Seoul, South Korea, 2008.

[13] E. Laroche, S. Kagami, Dynamical models for position measurement with global shutter and rolling shutter cameras, in: International Conference on Intelligent Robots and Systems, Saint Louis, MO, USA, 2009.

[14] E. Laroche, L. Cuvillon, S. Kagami, Hybrid kalman filter for improvement of camera-based position sensor, in: International Conference on Robotics and Automation, 2011, pp. 4405-4410.

[15] S. Hutchinson, G. D. Hager, P. I. Corke, A tutorial on visual servo control, IEEE Transactions on Robotics and Automation 12 (5) (1996) 651-670.

[16] A. Krupa, J. Gangloff, C. Doignon, M. de Mathelin, G. Morel, J. Leroy, L. Soler, Autonomous 3D positioning of surgical instruments in robotized laparoscopic surgery using visual servoing, IEEE Transactions on Robotics and Automation 19 (5) (2003) 842 - 853.

[17] J. Gangloff, M. de Mathelin, High speed visual servoing of a 6 DOF manipulator using multivariable predictive control, Advanced Robotics 17 (10) (2003) 993-1021.

[18] W. Bachta, P. Renaud, E. Laroche, A. Forgione, J. Gangloff, Active stabilization for robotized beating heart surgery, IEEE Transactions on Robotics 27 (4) (2011) 757-768. 
[19] J. Hoagg, S. Lacy, R. Erwin, D. Bernstein, First-order-hold sampling of positive real systems and subspace identification of positive real models, in: American Control Conference, Vol. 1, 2004, pp. 861-866.

[20] J. Takei, S. Kagami, K. Hashimoto, 3,000-fps 3-D shape measurement using a high-speed camera-projector system, in: International Conference on Intelligent Robots and Systems, 2007, pp. 3211-3216.

[21] D. Dudley, W. Duncan, J. Slaughter, Emerging digital micromirror device (DMD) applications, Proceedings of the International Society for Optics and Photonics 4985.

[22] R. Kalman, A new approach to linear filtering and prediction problems, ASME Journal of Basic Enginnering D 82 (1960) 34-45.

[23] R. Brown, P. Hwang, Introduction to Random Signals and Applied Kalman Filtering, 3rd Edition, Wiley and sons, 1997.

[24] M. Grewal, A. Andrews, Kalman Filtering: Theory and Practice Using MATLAB, 3rd Edition, Wiley and sons, 2008.

[25] R. Ginhoux, J. Gangloff, M. de Mathelin, L. Soler, M. A. Sanchez, J. Marescaux, Active filtering of physiological motion in robotized surgery using predictive control, IEEE Transactions on Robotics 21 (1) (2005) 67-79.

[26] J. Gagne, O. Piccin, E. Laroche, M. Diana, J. Gangloff, Gyrolock: Stabilizing the heart with control moment gyroscope (CMG) - from concept to first in vivo assessments, IEEE Transactions on Robotics 28 (4) (2012) $942-954$.

[27] W. Bachta, P. Renaud, L. Cuvillon, E. Laroche, A. Forgione, J. Gangloff, Motion prediction for computer-assisted beating heart surgery, IEEE Transactions on Biomedical Engineering 56 (11) (2009) 2551-2563.

[28] E. Yuen, P. Novotny, R. Howe, Quasiperiodic predictive filtering for robot-assisted beating heart surgery, in: International Conference on Robotics and Automation, 2008, pp. 3875-3880.

[29] R. Ginhoux, J. Gangloff, M. de Mathelin, L. Soler, M. Arenas Sanchez, J. Marescaux, Heart tracking in robotic surgery using $500 \mathrm{~Hz}$ visual 
servoing, model predictive control and an adaptive observer, in: International Conference on Robotics and Automation, Vol. 1, 2004, pp. 274-279. 


\section{Biographies}

Édouard Laroche graduated from the École Nationale Supérieure d'Électricité et de Mécanique (Nancy, France) in 1994. He received the Agrégation degree in electrical engineering and the Ph.D. from the École Normale Supérieure de Cachan (Paris, France) in 1995 and 2000, respectively. After being associate professor from 2000 to 2008, he has been full professor at the University of Strasbourg (France) since 2008 and is affiliated with the Laboratoire des Sciences de l'Ingénieur, de l'Informatique et de l'Imagerie. His research interests include robustness issues in identification and control of electromechanical systems.

Shingo Kagami received the B.E., M.E. and Ph.D. degrees in Mathematical Engineering and Information Physics from the University of Tokyo, Tokyo, Japan, in 1998, 2000, and 2003 respectively. He was a Research Fellow of Japan Science and Technology Corporation (JST) in 2003, and was a Research Associate of the University of Tokyo from 2003 to 2005. In 2005, he joined Graduate School of Information Sciences, Tohoku University, Sendai, Japan, where he is currently an Associate Professor. His research interests include systems, architectures and algorithms for high-speed vision processing and real-time sensory information processing.

Loïc Cuvillon graduated in control systems engineering from the École Nationale Supérieure de Physique de Strasbourg, France, in 2002, and the M.S. and Ph.D. degrees in robotics from Strasbourg University, France, in 2002 and 2006, respectively. Since 2007, he has been an Assistant Professor at Strasbourg University, where he is a member of the AVR (Control Vision and Robotics) team at the iCube laboratory. His current research interests include visual servoing of robotic manipulators, control of flexible structures, and medical robotics. 


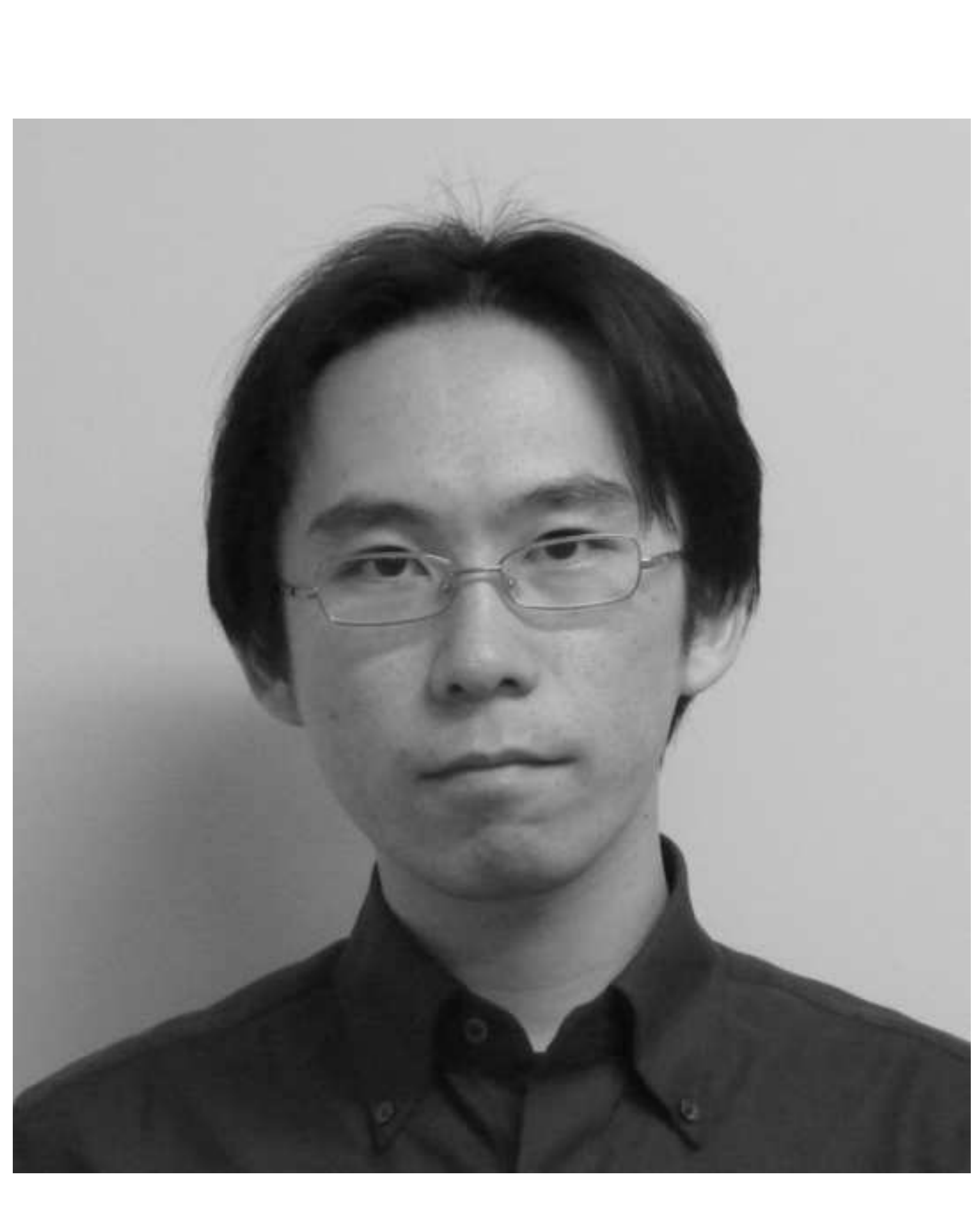

${ }^{*}$ Photo Shingo Kagami

mi
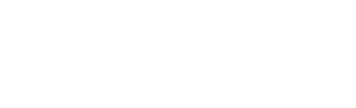

$x^{2}$

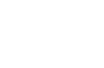
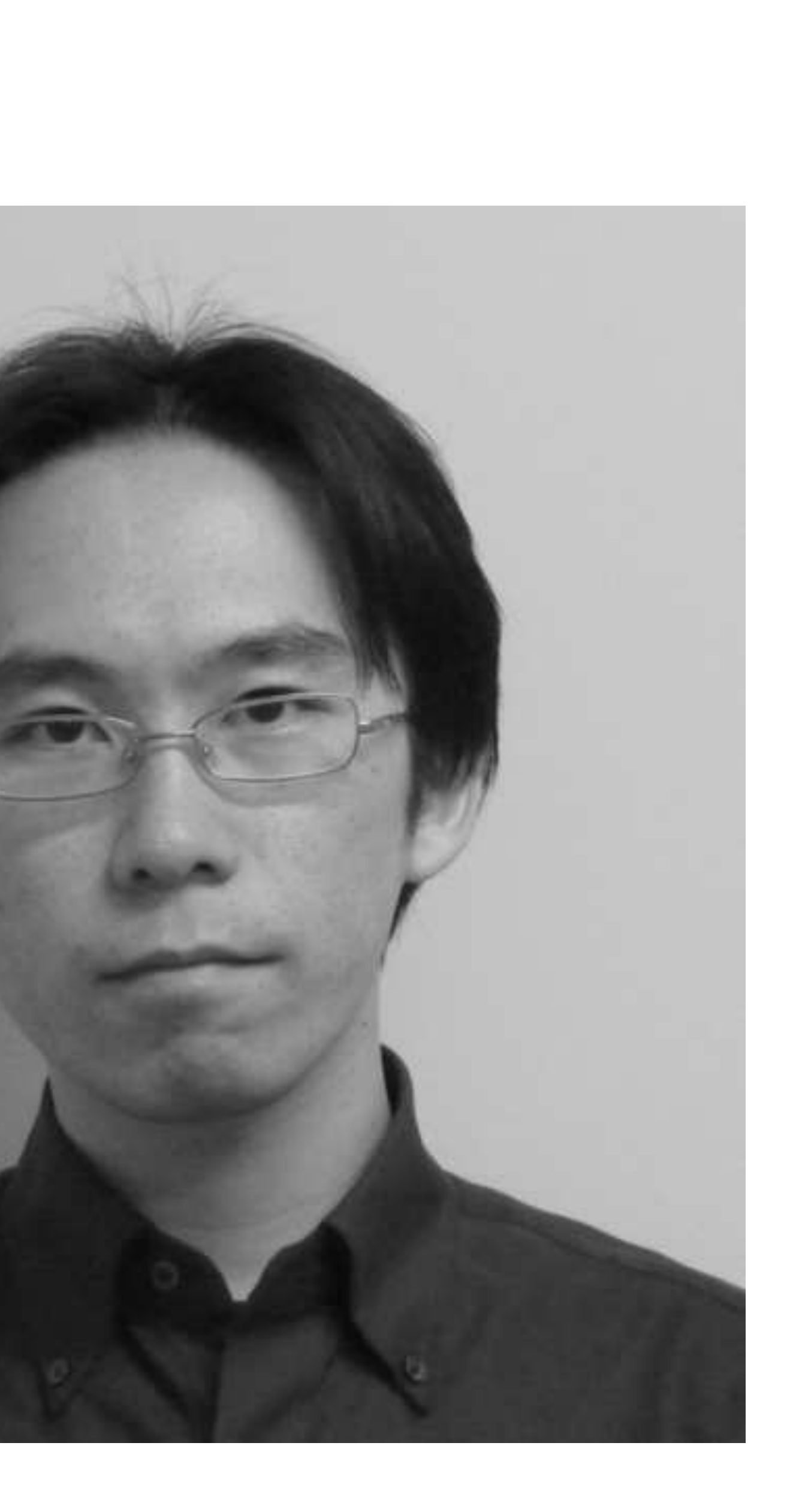


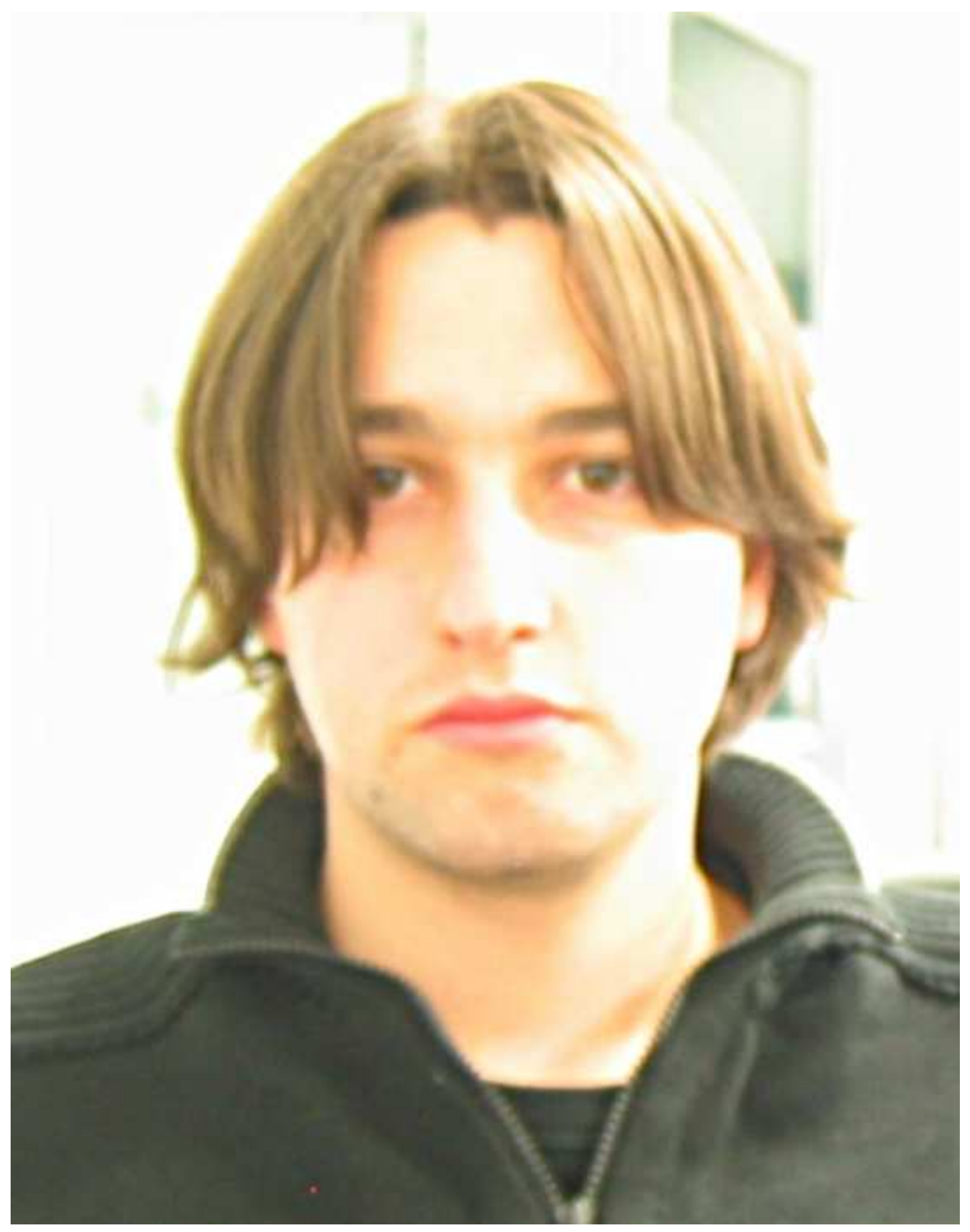

*Photo Loic Cuvillon 